\title{
Expected power-utility maximization under incomplete information and with Cox-process observations
}

\author{
Kazufumi Fujimoto* \\ The Bank of Tokyo-Mitsubishi UFJ, Ltd. \\ Corporate Risk Management Division, \\ Marunouchi 2-7-1, 100-8388, Chiyoda-ku,Tokyo, Japan \\ e-mail: m_fuji@kvj.biglobe.ne.jp \\ Hideo Nagai \\ Division of Mathematical Science for Social Systems, \\ Graduate School of Engineering Science, Osaka University, \\ Machikaneyama 1-3, 560-8531, Toyonaka, Osaka, Japan \\ e-mail: nagai@sigmath.es.osaka-u.ac.jp \\ Wolfgang J. Runggaldier \\ Dipartimento di Matematica Pura ed Applicata \\ Università di Padova, Via Trieste 63, I-35121-Padova \\ e-mail: runggal@math.unipd.it
}

\begin{abstract}
We consider the problem of maximization of expected terminal power utility (risk sensitive criterion). The underlying market model is a regime-switching diffusion model where the regime is determined by an unobservable factor process forming a finite state Markov process. The main novelty is due to the fact that prices are observed and the portfolio is rebalanced only at random times corresponding to a Cox process where the intensity is driven by the unobserved Markovian factor process as well. This leads to a more realistic modeling for many practical situations, like in markets with liquidity restrictions; on the other hand it considerably complicates the problem to the point that traditional methodologies cannot be directly applied. The approach presented here is specific to the power-utility. For log-utilities a different approach is presented in the companion paper [8].
\end{abstract}

Mathematics Subject Classification : Primary 93E20; Secondary 91B28, 93E11, 60J75.

Keywords : Portfolio optimization, Stochastic control, Incomplete information, Regime-switching models, Cox-process observations, Random trading times.

${ }^{*}$ The opinions expressed are those of the author and not those of The Bank of Tokyo-Mitsubishi UFJ. 


\section{Introduction}

In this paper we consider a classical portfolio optimization problem, namely the maximization of expected utility from terminal wealth. As criterion we take a variant of expected power utility maximization (the log utility function is studied in the companion paper [8]). More precisely we consider a risk-sensitive type criterion of the form

$$
\sup \frac{1}{\mu} E\left\{V_{T}^{\mu}\right\}
$$

where we restrict ourselves to the case of $\mu<0$ (concave utility). While the problem as such is a classical one, the novelty in this paper is given by a more realistic underlying market model, which implies that standard approaches such as Dynamic Programming and convex duality cannot be applied directly and a novel approach is required. Our market model is first of all of the regime-switching type where the factor process that specifies the regime may not be fully observable. This is still a relatively classical situation, for which one may use techniques from stochastic control under incomplete information. In fact, various papers have appeared in such a context and here we just mention some of the most recent ones that also summarize previous work in the area, more precisely [2], [16], [20]. The main novelty of our model is however given by the fact that the prices $S_{t}^{i}$, or equivalently their logarithmic values $X_{t}^{i}:=\log S_{t}^{i}$, of the risky assets in which one invests are supposed to be observable only at discrete random points in time $\tau_{0}, \tau_{1}, \tau_{2}, \cdots$, where the associated counting process is a Cox process (see e.g. [3], [11]) with intensity that depends on the same factor process that specifies the regime for the price evolution model. Such models are in fact relevant in financial applications since (see e.g. [7], [4], [17], [18]), especially on small time scales, prices do not vary continuously, but rather change and are observed only at discrete random points in time that correspond to the time instants when significant new information is absorbed by the market and/or market makers update their quotes. This setting leads to a stochastic control problem with incomplete information and observations given by a Cox process.

A classical approach to incomplete observation control problems is to first transform the problem into a so-called separated problem, where the unobservable part of the state is replaced by its conditional distribution. This requires to solve first the associated filtering problem, which already is non-standard and has been solved recently in [4] (see also [5]). Our major contribution here is on the control part of the separated problem that is approached in a non classical way. In particular we shall restrict the investment strategies to be rebalanced only at the random times $\tau_{k}$ where prices change. Although slightly less general from a theoretical point of view, restricting trading to discrete, in particular random times, is quite realistic in finance, where in practice one cannot rebalance a portfolio continuously: think of the case with transaction costs or with liquidity restrictions (in this latter context see e.g. [9], [10], [14], [17], [18], [19] where the authors consider illiquid markets, partly also with regime switching models as in this paper, but under complete information).

In the companion paper [8] we study the case of a log utility, for the solution of which one cannot simply carry over the approach that we develop here for the power-utility case, even if there are close analogies between the two approaches. In other words, for our nonclassical setup the approach that one has to use may depend on the specific case.

In section 2 we give a more precise definition of the model and of the investment strategy and, after recalling some of the main filtering results from [4], we also specify the objective function 
and the purpose of the study. Section 3 is intended to prepare for the main result of the paper stated in section 4. More precisely, subsection 3.1 consists mainly in estimation results and in establishing continuity properties, while subsections 3.2 and 3.3 contain results that will be used to obtain an approximation, of the type of "value iteration", of the optimal value function and a Dynamic Programming principle that is specific to the given problem setting. These results serve also the purpose of obtaining a methodology to determine an optimal strategy. An Appendix contains auxiliary technical results.

\section{Market model, basic tools and objective}

\subsection{Introductory remarks}

As mentioned in the general Introduction, we consider here the problem of maximization of expected power-utility from terminal wealth, when the dynamics of the prices of the risky assets in which one invests are of the usual diffusion type but with the coefficients in the dynamics depending on an unobservable finite-state Markovian factor process (regime-switching model). In addition it is assumed that the risky asset prices $S_{t}^{i}$, or equivalently their logarithmic values $X_{t}^{i}:=\log S_{t}^{i}$, are observed only at random times $\tau_{0}, \tau_{1}, \cdots$ for which the associated counting process forms a Cox process with an intensity $n\left(\theta_{t}\right)$ that also depends on the unobservable factor process $\theta_{t}$.

\subsection{The market model}

Let $\theta_{t}$ be the hidden finite state Markovian factor process. With $Q$ denoting its transition intensity matrix ( $Q$-matrix) its dynamics are given by

$$
d \theta_{t}=Q^{*} \theta_{t} d t+d M_{t}, \theta_{0}=\xi
$$

where $M_{t}$ is a jump-martingale on a given filtered probability space $\left(\Omega, \mathcal{F}, \mathcal{F}_{t}, P\right)$. If $N$ is the number of possible values of $\theta_{t}$, we may without loss of generality take as its state space the set $E=\left\{e_{1}, \ldots, e_{N}\right\}$, where $e_{i}$ is a unit vector for each $i=1, \ldots, N$ (see $[6])$.

The evolution of $\theta_{t}$ may also be characterized by the process $\pi_{t}$ given by the state probability vector that takes values in the set

$$
\mathcal{S}_{N}:=\left\{\pi \in \mathbb{R}^{N} \mid \sum_{i=1}^{N} \pi^{i}=1,0 \leq \pi^{i} \leq 1, i=1,2, \ldots, N\right\}
$$

namely the set of all probability measures on $E$ and we have $\pi_{0}^{i}=P\left(\xi=e_{i}\right)$. Denoting by $\mathcal{M}(E)$ the set of all finite nonnegative measures on $E$, it follows that $\mathcal{S}_{N} \subset \mathcal{M}(E)$. In our study it will be convenient to consider on $\mathcal{M}(E)$ the Hilbert metric $d_{H}(\pi, \bar{\pi})$ defined (see [1] [12] [13]) by

$$
d_{H}(\pi, \bar{\pi}):=\log \left(\sup _{\bar{\pi}(A)>0, A \subset E} \frac{\pi(A)}{\bar{\pi}(A)} \sup _{\pi(A)>0, A \subset E} \frac{\bar{\pi}(A)}{\pi(A)}\right)
$$


Notice that, while $d_{H}$ is only a pseudo-metric on $\mathcal{M}(E)$, it is a metric on $\mathcal{S}_{N}([1])$. Furthermore, see Lemma 3.4 in [12], for $\forall \pi, \bar{\pi} \in \mathcal{S}_{N}$ we also have

$$
\|\pi-\bar{\pi}\|_{T V} \leq \frac{2}{\log 3} d_{H}(\pi, \bar{\pi}),
$$

where $\|\cdot\|_{T V}$ is the total variation on $\mathcal{S}_{N}$.

In our market we consider $m$ risky assets, for which the price processes $S^{i}=\left(S_{t}^{i}\right)_{t \geq 0}, i=$ $1, \ldots, m$ are supposed to satisfy

$$
d S_{t}^{i}=S_{t}^{i}\left\{r^{i}\left(\theta_{t}\right) d t+\sum_{j} \sigma_{j}^{i}\left(\theta_{t}\right) d B_{t}^{j}\right\}
$$

for given coefficients $r^{i}(\theta)$ and $\sigma_{j}^{i}(\theta)$ and with $B_{t}^{j}(j=1, \cdots, m)$ independent $\left(\mathcal{F}_{t}, P\right)$-Wiener processes. Letting $X_{t}:=\log S_{t}$, by Itô's formula we have

$$
X_{t}=X_{0}+\int_{0}^{t} r\left(\theta_{s}\right)-d\left(\sigma \sigma^{*}\left(\theta_{s}\right)\right) d s+\int_{0}^{t} \sigma\left(\theta_{s}\right) d B_{s}
$$

where by $d\left(\sigma \sigma^{*}(\theta)\right)$ we denote the diagonal matrix $d\left(\sigma \sigma^{*}(\theta)\right)$ := $\left(\frac{1}{2}\left(\sigma \sigma^{*}\right)^{11}(\theta), \ldots, \frac{1}{2}\left(\sigma \sigma^{*}\right)^{m m}(\theta)\right)$. As usual there is also a locally non-risky asset (bond) with price $S_{t}^{0}$ satisfying

$$
d S_{t}^{0}=r_{0} S_{t}^{0} d t
$$

where $r_{0}$ stands for the short rate of interest.

As already mentioned, the asset prices and thus also their logarithms are observed only at random times $\tau_{0}, \tau_{1}, \tau_{2}, \ldots$ and we shall put $X_{k}=\left(X_{k}^{1}, \ldots, X_{k}^{m}\right)$ with $X_{k}^{i}:=X_{\tau_{k}}^{i},(i=$ $1, \cdots, m ; k \in \mathbb{N})$. The observations are thus given by the sequence $\left(\tau_{k}, X_{k}\right)_{k \in \mathbb{N}}$ that forms a multivariate marked point process with counting measure

$$
\mu(d t, d x)=\sum_{k} \mathbf{1}_{\left\{\tau_{k}<\infty\right\}} \delta_{\left\{\tau_{k}, X_{k}\right\}}(t, x) d t d x .
$$

The corresponding counting process $\Lambda_{t}:=\int_{0}^{t} \int_{\mathbb{R}^{m}} \mu(d t, d x)$ is supposed to be a Cox process with intensity $n\left(\theta_{t}\right)$, i.e. $\Lambda_{t}-\int_{0}^{t} n\left(\theta_{s}\right) d s$ is an $\left(\mathcal{F}_{t}, P\right)-$ martingale. We consider two sub-filtrations related to $\left(\tau_{k}, X_{k}\right)_{k \in \mathbb{N}}$ namely

$$
\begin{aligned}
\mathcal{G}_{t} & :=\mathcal{F}_{0} \vee \sigma\left\{\mu((0, s] \times B): s \leq t, B \in \mathcal{B}\left(\mathbb{R}^{m}\right)\right\}, \\
\mathcal{G}_{k} & :=\mathcal{F}_{0} \vee \sigma\left\{\tau_{0}, X_{0}, \tau_{1}, X_{1}, \tau_{2}, X_{2}, \ldots, \tau_{k}, X_{k}\right\} .
\end{aligned}
$$

In our development below we shall often make use of the following notations. Let

$$
\begin{aligned}
m_{0}(\theta) & :=r_{0}, \sigma_{0}(\theta):=0 \\
m_{i}(\theta) & :=r^{i}(\theta)-\frac{1}{2}\left(\sigma \sigma^{*}\right)^{i i}(\theta) \text { for } i=1, \ldots, m \\
\sigma_{i}(\theta) & :=\sqrt{\left(\sigma \sigma^{*}\right)^{i i}(\theta)} \text { for } i=1, \ldots, m
\end{aligned}
$$


and put $m(\theta):=\left\{m_{1}(\theta), \cdots, m_{m}(\theta)\right\}$. For the conditional (on $\mathcal{F}^{\theta}$ ) mean and variance of $X_{s}-$ $X_{t}=\left\{X_{s}^{i}-X_{t}^{i}\right\}_{i=1, \ldots, m}$ with $t<s \leq T$ we then have

$$
m_{t, s}^{\theta}=\int_{t}^{s} m\left(\theta_{u}\right) d u \quad, \quad \sigma_{t, s}^{\theta}=\int_{t}^{s} \sigma \sigma^{*}\left(\theta_{u}\right) d u
$$

and, for $z \in \mathbb{R}^{m}$ and $t<s \leq T$, we set

$$
\rho_{t, s}^{\theta}(z) \sim N\left(z ; m_{t, s}^{\theta}, \sigma_{t, s}^{\theta}\right)
$$

namely the joint conditional (on $\mathcal{F}^{\theta}$ ) $m$-dimensional normal density function of $X_{s}-X_{t}$ with mean vector $m_{t, s}^{\theta}$ and covariance matrix $\sigma_{t, s}^{\theta}$. Notice that, although here and in the companion paper [8] we use the same symbol $\rho_{\text {. }}^{\theta}(z)$, the corresponding quantities are different in the two papers due to a different mean value which is a consequence of the fact that here we base ourselves on the process $X_{t}=\log S_{t}$ while in [8] it is $\tilde{X}_{t}=\log \tilde{S}_{t}$.

\subsection{Investment strategies and portfolios}

As mentioned in the Introduction, since observations take place at random time points $\tau_{k}$, we shall consider investment strategies that are rebalanced only at those same time points $\tau_{k}$.

Let $N_{t}^{i}$ be the number of assets of type $i$ held in the portfolio at time $t, N_{t}^{i}=\sum_{k} \mathbf{1}_{\left[\tau_{k}, \tau_{k+1}\right)}(t) N_{\tau_{k}}^{i}$. The wealth process is defined by

$$
V_{t}:=\sum_{i=0}^{m} N_{t}^{i} S_{t}^{i}
$$

Consider then the investment ratios

$$
h_{t}^{i}:=\frac{N_{t}^{i} S_{t}^{i}}{V_{t}}, \quad i=1, \cdots, m
$$

and set $h_{k}^{i}:=h_{\tau_{k}}^{i}$. The set of admissible investment ratios is given by

$$
\bar{H}_{m}:=\left\{\left(h^{1}, \ldots, h^{m}\right) ; h^{1}+h^{2}+\ldots+h^{m} \leq 1,0 \leq h^{i}, i=1,2, \ldots, m\right\},
$$

i.e. shortselling is not allowed and notice that $\bar{H}_{m}$ is bounded and closed. Put $h=\left(h^{1}, \cdots, h^{m}\right)$. Analogously to [15] define next a function $\gamma: \mathbb{R}^{m} \times \bar{H}_{m} \rightarrow \bar{H}_{m}$ by

$$
\gamma^{i}(z, h):=\frac{h^{i} \exp \left(z^{i}\right)}{1+\sum_{i=1}^{m} h^{i}\left(\exp \left(z^{i}\right)-1\right)} i=1, \ldots, \ldots .
$$

Putting $\tilde{X}_{t}^{i}:=\log \tilde{S}_{t}^{i}$ with $\tilde{S}_{t}^{i}:=\frac{S_{t}^{i}}{S_{t}^{0}}$ (discounted asset prices) and noticing that $N_{t}$ is constant on $\left[\tau_{k}, \tau_{k+1}\right)$, for $i=1, \ldots, m$, and $t \in\left[\tau_{k}, \tau_{k+1}\right)$, let

$$
\begin{aligned}
h_{t}^{i} & =\frac{N_{t}^{i} S_{t}^{i}}{\sum_{i=0}^{m} N_{t}^{i} S_{t}^{i}}=\frac{N_{k}^{i} S_{t}^{i}}{\sum_{i=0}^{m} N_{k}^{i} S_{t}^{i}} \\
& =\frac{N_{k}^{i} S_{k}^{i} S_{t}^{i} / S_{k}^{i}}{\sum_{i=0}^{m} N_{k}^{i} S_{k}^{i} S_{t}^{i} / S_{k}^{i}}=\frac{h_{k}^{i} S_{t}^{i} / S_{k}^{i}}{\sum_{i=0}^{m} h_{k}^{i} S_{t}^{i} / S_{k}^{i}}=\frac{h_{k}^{i} S_{k}^{0} / S_{t}^{0} S_{t}^{i} / S_{k}^{i}}{\sum_{i=0}^{m} h_{k}^{i} S_{k}^{0} / S_{t}^{0} S_{t}^{i} / S_{k}^{i}} \\
& =\frac{h_{k}^{i} \exp \left(\tilde{X}_{t}^{i}-\tilde{X}_{k}^{i}\right)}{h_{k}^{0}+\sum_{i=1}^{m} h_{k}^{i} \exp \left(\tilde{X}_{t}^{i}-\tilde{X}_{k}^{i}\right)}=\frac{h_{k}^{i} \exp \left(\tilde{X}_{t}^{i}-\tilde{X}_{k}^{i}\right)}{1+\sum_{i=1}^{m} h_{k}^{i}\left(\exp \left(\tilde{X}_{t}^{i}-\tilde{X}_{k}^{i}\right)-1\right)} \\
& =\gamma^{i}\left(\tilde{X}_{t}-\tilde{X}_{k}, h_{k}\right) .
\end{aligned}
$$


The set of admissible strategies $\mathcal{A}$ is defined by

$$
\mathcal{A}:=\left\{\left\{h_{k}\right\}_{k=0}^{\infty} \mid h_{k} \in \bar{H}_{m}, \mathcal{G}_{k} \text { m'ble for all } k \geq 0\right\} .
$$

Furthermore, for $n>0$, we let

$$
\mathcal{A}^{n}:=\left\{h \in \mathcal{A} \mid h_{n+i}=h_{\tau_{n+i}-} \text { for all } i \geq 1\right\} .
$$

Notice that, by the definition of $\mathcal{A}^{n}$, for all $k \geq 1, h \in \mathcal{A}^{n}$ we have

$$
\begin{gathered}
h_{n+k}^{i}=h_{\tau_{n+k}-}^{i} \\
\Leftrightarrow \frac{N_{n+k}^{i} S_{n+k}^{i}}{\sum_{i=0}^{m} N_{n+k}^{i} S_{n+k}^{i}}=\frac{N_{n+k-1}^{i} S_{n+k}^{i}}{\sum_{i=0}^{m} N_{n+k}^{i} S_{n+k}^{i}} \\
\Leftrightarrow N_{n+k}=N_{n+k-1} .
\end{gathered}
$$

Therefore, for $k \geq 1$

$$
N_{n+k}=N_{n}
$$

and

$$
\mathcal{A}^{0} \subset \mathcal{A}^{1} \subset \cdots \mathcal{A}^{n} \subset \mathcal{A}^{n+1} \cdots \subset \mathcal{A} .
$$

Remark 2.1. Notice that, for a given finite sequence of investment ratios $h_{0}, h_{1}, \cdots, h_{n}$ such that $h_{k}$ is an $\mathcal{G}_{k}-$ measurable, $\bar{H}_{m}$-valued random variable for $k \leq n$, there exists $h^{(n)} \in \mathcal{A}^{n}$ such that $h_{k}^{(n)}=h_{k}, k=0, \cdots, n$. Indeed, if $N_{t}$ is constant on $\left[\tau_{n}, T\right)$, then for $h_{t}$ we have $h_{t}=\gamma\left(\tilde{X}_{t}-\tilde{X}_{n}, h_{n}\right), \forall t \geq \tau_{n}$. Therefore, by setting $h_{\ell}^{(n)}=h_{\ell}, \ell=0, \cdots, n$, and $h_{n+k}^{(n)}=$ $h_{\tau_{n}+k}, k=1,2, \cdots$, since the vector process $S_{t}$ and the vector function $\gamma\left(\cdot, h_{n}\right)$ are continuous, we see that $h_{n+k}^{(n)}=h_{\tau_{n}+k^{-}}, k=1,2, \cdots$.

Finally, considering only self-financing portfolios, for their value process we have the dynamics

$$
\frac{d V_{t}}{V_{t}}=\left[r_{0}+h_{t}^{*}\left\{r\left(\theta_{t}\right)-r_{0} \mathbf{1}\right\}\right] d t+h_{t}^{*} \sigma\left(\theta_{t}\right) d B_{t} .
$$

\section{$2.4 \quad$ Filtering}

As mentioned in the Introduction, the usual approach to stochastic control problems under incomplete information is to first transform them into a so-called separated problem, where the unobservable part of the state is replaced by its conditional (filter) distribution. This implies that we first have to study this conditional distribution and its (Markovian) dynamics, i.e. we have to study the associated filtering problem.

The filtering problem for our specific case, where the observations are given by a Cox process with intensity expressed as a function of the unobserved state, has been studied in [4] (see also [5]). Here we briefly summarize the main results from [4] in view of their use in our control problem.

Recalling the definition of $\rho^{\theta}(z)$ in $(2.12)$ and putting

$$
\phi^{\theta}\left(\tau_{k}, t\right)=n\left(\theta_{t}\right) \exp \left(-\int_{\tau_{k}}^{t} n\left(\theta_{s}\right) d s\right)
$$


for a given function $f(\theta)$ we let

$$
\begin{gathered}
\psi_{k}(f ; t, x):=E\left[f\left(\theta_{t}\right) \rho_{\tau_{k}, t}^{\theta}\left(x-X_{k}\right) \phi^{\theta}\left(\tau_{k}, t\right) \mid \sigma\left\{\theta_{\tau_{k}}\right\} \vee \mathcal{G}_{k}\right] \\
\bar{\psi}_{k}(f ; t):=\int \psi_{k}(f ; t, x) d x=E\left[f\left(\theta_{t}\right) \phi^{\theta}\left(\tau_{k}, t\right) \mid \sigma\left\{\theta_{\tau_{k}}\right\} \vee \mathcal{G}_{k}\right] \\
\pi_{t}(f)=E\left[f\left(\theta_{t}\right) \mid \mathcal{G}_{t}\right]
\end{gathered}
$$

with ensuing obvious meanings of $\pi_{\tau_{k}}\left(\psi_{k}(f ; t, y)\right)$ and $\pi_{\tau_{k}}\left(\bar{\psi}_{k}(f ; t)\right)$ where we consider $\psi_{k}(f ; t, y)$ and $\bar{\psi}_{k}(f ; t)$ as functions of $\theta_{\tau_{k}}$. The process $\pi_{t}(f)$ is called the filter process for $f\left(\theta_{t}\right)$.

We have (see Lemma 4.1 in [4])

Lemma 2.1. The compensator of the random measure $\mu(d t, d y)$ in (2.8) on the $\sigma$-algebra $\tilde{\mathcal{P}}(\mathcal{G})=\mathcal{P}(\mathcal{G}) \otimes \mathcal{B}\left(R^{m}\right)$ with $\mathcal{P}(\mathcal{G})$ the predictable $\sigma$-algebra on $\Omega \times[0, \infty)$, is given by the following nonnegative random measure

$$
\nu(d t, d y)=\sum_{k} \mathbf{1}_{\left(\tau_{k}, \tau_{k+1}\right]}(t) \frac{\pi_{\tau_{k}}\left(\psi_{k}(1, t, y)\right)}{\int_{t}^{\infty} \pi_{\tau_{k}}\left(\bar{\psi}_{k}(1, s)\right) d s} d t d y
$$

The main filtering result is the following (see Theorem 4.1 in [4]).

Theorem 2.1. For any bounded function $f(\theta)$, the differential of the optimal filter $\pi_{t}(f)$ is given by

$$
\begin{aligned}
d \pi_{t}(f)= & \pi_{t}(L f) d t \\
& +\int \sum_{k} \mathbf{1}_{\left(\tau_{k}, \tau_{k+1}\right]}(t)\left[\frac{\pi_{\tau_{k}}\left(\psi_{k}(f ; t, y)\right)}{\pi_{\tau_{k}}\left(\psi_{k}(1 ; t, y)\right)}-\pi_{t-}(f)\right](\mu-\nu)(d t, d y),
\end{aligned}
$$

where $L$ is the generator of the Markov process $\theta_{t}($ namely $L=Q)$.

Corollary 2.1. We have

$$
\pi_{\tau_{k+1}}(f)=\left.\frac{\pi_{\tau_{k}}\left(\psi_{k}(f ; t, x)\right)}{\pi_{\tau_{k}}\left(\psi_{k}(1 ; t, x)\right)}\right|_{t=\tau_{k+1}, x=X_{k+1}}
$$

Recall that in our setting $\theta_{t}$ is an $N$-state Markov chain with state space $E=\left\{e_{1}, \ldots, e_{N}\right\}$, where $e_{i}$ is a unit vector for each $i=1, \ldots, N$. One may then write $f\left(\theta_{t}\right)=f\left(e_{i}\right) \mathbf{1}_{e_{i}}\left(\theta_{t}\right)$. For $i=1, \ldots, N$ let $\pi_{t}^{i}=\pi_{t}\left(\mathbf{1}_{e_{i}}\left(\theta_{t}\right)\right)$ and

$$
\begin{gathered}
r_{j i}(t, z):=E\left[\exp \left(\int_{0}^{t}-n\left(\theta_{s}\right) d s\right) \rho_{0, t}^{\theta}(z) \mid \theta_{0}=e_{j}, \theta_{t}=e_{i}\right], \\
p_{j i}(t):=P\left(\theta_{t}=e_{i} \mid \theta_{0}=e_{j}\right)
\end{gathered}
$$

and, noticing that $\pi_{t} \in \mathcal{S}_{N}$, define the function $M:[0, \infty) \times \mathbb{R}^{m} \times \mathcal{S}_{N} \rightarrow \mathcal{S}_{N}$ by

$$
\begin{gathered}
M^{i}(t, x, \pi):=\frac{\sum_{j} n\left(e_{i}\right) r_{j i}(t, x) p_{j i}(t) \pi^{j}}{\sum_{i j} n\left(e_{i}\right) r_{j i}(t, x) p_{j i}(t) \pi^{j}} \\
M(t, x, \pi):=\left(M^{1}(t, x, \pi), M^{2}(t, x, \pi), \ldots, M^{N}(t, x, \pi)\right) .
\end{gathered}
$$


For $A \subset E$

$$
M(t, x, \pi)(A):=\sum_{i=1}^{N} M^{i}(t, x, \pi) 1_{\left\{e_{i} \in A\right\}} .
$$

The following corollary, for the proof of which we refer to the companion paper [8] (see also [4]), describes the dynamics of the filter process.

Corollary 2.2. For the generic $i$-th state one has

$$
\pi_{k+1}^{i}=M^{i}\left(\tau_{k+1}-\tau_{k}, X_{k+1}-X_{k}, \pi_{k}\right)
$$

and the process $\left\{\tau_{k}, \pi_{k}, X_{k}\right\}_{k=1}^{\infty}$ is a Markov process with respect to $\mathcal{G}_{k}$.

Finally from results in [1], [12] and [13] it follows (see again the companion paper [8]) that

$$
d_{H}(M(t, x, \pi), M(t, x, \bar{\pi})) \leq d_{H}(\pi, \bar{\pi}) .
$$

\subsection{Objective and purpose of the study}

Given a finite planning horizon $T>0$, our problem of maximization of expected terminal power utility consists in determining

$$
\begin{aligned}
& \sup _{h \in \mathcal{A}} \frac{1}{\mu} \log E\left[V_{T}^{\mu} \mid \tau_{0}=0, \pi_{0}=\pi\right] \\
& \quad=\log v_{0}+\sup _{h \in \mathcal{A}} \frac{1}{\mu} \log E\left[\frac{V_{T}^{\mu}}{V_{0}^{\mu}} \mid \tau_{0}=0, \pi_{0}=\pi\right],
\end{aligned}
$$

for $\mu<0$ as well as an optimal maximizing strategy $\hat{h} \in \mathcal{A}$.

Notice that, in order to study the optimization problem (2.34), it suffices to analyze the criterion function

$$
W(t, \pi, h .):=\frac{1}{\mu} \log E\left[\frac{V_{T}^{\mu}}{V_{t}^{\mu}} \mid \tau_{0}=t, \pi_{0}=\pi\right] .
$$

The optimal value function will then be defined as

$$
W(t, \pi):=\sup _{h \in \mathcal{A}} W(t, \pi, h \cdot) .
$$

The main result of this paper, stated and proved in Theorem 4.1 in section 4, consists basically of two parts:

- An approximation theorem stating that a sequence $\bar{W}^{n}(t, \pi)$ of value functions, obtained by a value-iteration type algorithm (see (3.27) below), approximates arbitrarily closely the optimal value function $W(t, x)$. This then leads also to the optimal strategy.

- A Dynamic Programming type relation for the optimal value function. 
To prepare for the proof of the main result, in the next section 3 we shall introduce some relevant notions and quantities and prove various preliminary results. More specifically, in subsection 3.1 we shall, in addition to giving some basic definitions, prove various estimates together with continuity properties. In subsection 3.2 we then derive some approximation results that are preliminary to the main approximation result in Theorem 4.1 and in subsection 3.3 we add some limiting results that will allow us to complete the main approximation result and to prepare also for the second result, namely the Dynamic Programming relation.

\section{Preliminary analysis}

As already mentioned, in this section we shall introduce relevant notions and quantities and prove various preliminary results, divided into three subsections.

In the sequel we shall for simplicity use mostly the shorthand notation

$$
E^{t, \pi}[\cdot] \equiv E\left[\cdot \mid \tau_{0}=t, \pi_{0}=\pi\right]
$$

and also use the following notations

$$
\left\{\begin{array}{l}
\bar{m}:=\max _{0 \leq i \leq m} \max _{1 \leq j \leq N} m_{i}\left(e_{j}\right) \\
\underline{m}:=\min _{0 \leq i \leq m} \min _{1 \leq j \leq N} m_{i}\left(e_{j}\right) \wedge 0 \quad \text { implying that } \underline{m} \leq 0 \\
\bar{\sigma}:=\max _{0 \leq i \leq m} \max _{1 \leq j \leq N} \sigma_{i}\left(e_{j}\right) \\
l(t):=E\left[\left|1-\exp \left(\mu\left|X_{t}-X_{0}\right|\right)\right|\right] \\
c:=E\left[1_{\left\{\tau_{1} \leq T\right\}}\right] \\
\underline{n}:=\min n(\theta)=\min _{i} n\left(e_{i}\right) \\
\bar{n}:=\max n(\theta)=\max _{i} n\left(e_{i}\right)
\end{array}\right.
$$

\subsection{Basic estimates}

We start from the following representation of the criterion function.

Lemma 3.1. For $t \in[0, T], \pi \in \mathcal{S}_{N}$

$$
\begin{aligned}
& W(t, \pi, h .) \\
= & \frac{1}{\mu} \log E^{t, \pi}\left[\exp \left(\mu \sum_{k=1}^{\infty} D\left(h_{k-1}, X_{T \wedge \tau_{k}}-X_{\tau_{k-1}}\right) 1_{\left\{\tau_{k-1}<T\right\}}\right)\right],
\end{aligned}
$$

where,

$$
D(h, x):=\log \left(\sum_{i=0}^{m} h^{i} \exp \left(x^{i}\right)\right) .
$$


Proof. Since $\sum_{i=0}^{m} h^{i}=1$,

$$
D(h, 0)=\log \left(\sum_{i=0}^{m} h^{i}\right)=0
$$

for $h \in \bar{H}_{m}$. For $k \geq 0, T \in\left[\tau_{k}, \tau_{k+1}\right]$

$$
\begin{aligned}
\frac{V_{T}^{\mu}}{V_{k}^{\mu}} & =\left(\sum_{i=0}^{m} \frac{N_{T}^{i} S_{T}^{i}}{V_{k}}\right)^{\mu}=\left(\sum_{i=0}^{m} \frac{N_{k}^{i} S_{k}^{i}}{V_{k}} \frac{S_{T}^{i}}{S_{k}^{i}}\right)^{\mu}=\left(\sum_{i=0}^{m} h_{k}^{i} \frac{S_{T}^{i}}{S_{k}^{i}}\right)^{\mu} \\
& =\left(\sum_{i=0}^{m} h_{k}^{i} \exp \left(X_{T}^{i}-X_{k}^{i}\right)\right)^{\mu} \\
& =\exp \left(\mu D\left(h_{k}, X_{T}-X_{k}\right)\right) .
\end{aligned}
$$

For $k \geq 1, T<\tau_{k}$

$$
\frac{V_{T \wedge \tau_{k+1}}^{\mu}}{V_{T \wedge \tau_{k}}^{\mu}}=\frac{V_{T}^{\mu}}{V_{T}^{\mu}}=1,
$$

and

$$
\exp \left(\mu D\left(h_{k}, X_{T \wedge \tau_{k+1}}-X_{T \wedge \tau_{k}}\right)\right)=\exp \left(\mu D\left(h_{k}, X_{T}-X_{T}\right)\right)=1
$$

Therefore, we obtain

$$
\begin{aligned}
E^{t, \pi}\left[\left(V_{T} / V_{t}\right)^{\mu}\right] & =E^{t, \pi}\left[\prod_{k=1}^{\infty} \frac{V_{T \wedge \tau_{k}}^{\mu}}{\left.V_{T \wedge \tau_{k-1}}^{\mu}\right]}\right. \\
& =E^{t, \pi}\left[\exp \left(\mu \sum_{k=1}^{\infty} D\left(h_{k-1}, X_{T \wedge \tau_{k}}-X_{T \wedge \tau_{k-1}}\right)\right)\right] \\
& =E^{t, \pi}\left[\exp \left(\mu \sum_{k=1}^{\infty} D\left(h_{k-1}, X_{T \wedge \tau_{k}}-X_{\tau_{k-1}}\right) 1_{\left\{\tau_{k-1}<T\right\}}\right)\right] .
\end{aligned}
$$

The representation of $W(t, \pi, h$. $)$ in Lemma 3.1 leads us to define a function that will play a crucial role in the sequel, namely

Definition 3.1. Let the function $\bar{W}^{0}(t, \pi, h)$ be defined as

$$
\bar{W}^{0}(t, \pi, h):=\frac{1}{\mu} \log E^{t, \pi}\left[\exp \left(\mu D\left(h, X_{T}-X_{t}\right)\right)\right] .
$$

For the function $\bar{W}^{0}(t, \pi, h)$ in the above Definition 3.1 we now obtain estimation and continuity results as stated in the following proposition.

Proposition 3.1. For $t \in[0, T], h \in \bar{H}_{m}$, we have the estimate:

$$
\exp \left(\left(\mu \bar{m}+\frac{\mu \bar{\sigma}^{2}}{2}\right)(T-t)\right) \leq E^{t, \pi}\left[\exp \left(\mu D\left(h, X_{T}-X_{t}\right)\right)\right] \leq \exp \left(\left(\mu \underline{m}+\frac{(\mu \bar{\sigma})^{2}}{2}\right)(T-t)\right),
$$


from which it immediately follows that

$$
\left(\underline{m}+\frac{\mu \bar{\sigma}^{2}}{2}\right)(T-t) \leq \bar{W}^{0}(t, \pi, h) \leq\left(\bar{m}+\frac{\bar{\sigma}^{2}}{2}\right)(T-t) .
$$

Furthermore, $\bar{W}^{0}(t, \pi, h)$ is a continuous function on $[0, T] \times \mathcal{S}_{N} \times \bar{H}_{m}$ and the following estimates hold:

$$
\begin{gathered}
\left|\exp \left(\mu \bar{W}^{0}(t, \pi, h)\right)-\exp \left(\mu \bar{W}^{0}(t, \bar{\pi}, h)\right)\right| \leq \exp \left(\left(\mu \underline{m}+\frac{(\mu \bar{\sigma})^{2}}{2}\right)(T-t)\right) \frac{2}{\log 3} d_{H}(\pi, \bar{\pi}) \\
\left|\exp \left(\mu \bar{W}^{0}(t, \pi, h)\right)-\exp \left(\mu \bar{W}^{0}(\bar{t}, \pi, h)\right)\right| \leq \exp \left(\left(\mu \underline{m}+\frac{(\mu \bar{\sigma})^{2}}{2}\right)(T-t)\right) l(t-\bar{t}) \text { for } \bar{t}<t
\end{gathered}
$$

where $d_{H}$ was defined in (2.3).

Proof. See the Appendix.

We shall now introduce basic quantities that we shall use systematically throughout. The first one concerns useful function spaces, namely

Definition 3.2. By $\mathcal{G}$ we denote the function space

$$
\left.\mathcal{G}:=\left\{g \in C\left([0, T] \times \mathcal{S}_{N}\right) \mid\left(\underline{m}+\frac{\mu \bar{\sigma}^{2}}{2}\right)(T-t) \leq g(t, \pi) \leq\left(\bar{m}+\frac{\bar{\sigma}^{2}}{2}\right)(T-t)\right)\right\} .
$$

Furthermore, we let $\mathcal{G}_{1} \subset \mathcal{G}$ be the subspace

$$
\mathcal{G}_{1}:=\left\{g \in \mathcal{G}|| \exp (\mu g(t, \pi))-\exp (\mu g(t, \bar{\pi})) \mid \leq \frac{2}{\log 3} d_{H}(\pi, \bar{\pi}) \exp \left(\left(\mu \underline{m}+\frac{(\mu \bar{\sigma})^{2}}{2}\right)(T-t)\right)\right\}
$$

and $\mathcal{G}_{2} \subset \mathcal{G}$ be the subspace of functions $g \in \mathcal{G}$ satisfying, for $\bar{t}<t$,

$$
|\exp (\mu g(t, \pi))-\exp (\mu g(\bar{t}, \pi))| \leq k(t-\bar{t}) \exp \left(\left(\mu \underline{m}+\frac{(\mu \bar{\sigma})^{2}}{2}\right)(T-t)\right),
$$

where $k(t)$ is some nonnegative function on $\mathbb{R}$ such that $k(0)=0$ and continuous at 0 .

The second one concerns a crucial auxiliary quantity that will play a major role in the proofs to follow, namely

Definition 3.3. For each $g \in \mathcal{G}$ let $\hat{\xi}:[0, T] \times \mathcal{S}_{N} \times \bar{H}_{m} \rightarrow \mathbb{R}$ be the function defined by

$$
\hat{\xi}(t, \pi, h ; g):=\frac{1}{\mu} \log E^{t, \pi}\left[\exp \left(\mu D\left(h, X_{T \wedge \tau_{1}}-X_{t}\right)+\mu 1_{\left\{\tau_{1}<T\right\}} g\left(\tau_{1}, \pi_{1}\right)\right)\right] .
$$

We can now state and prove the following estimation result

Proposition 3.2. For each $g \in \mathcal{G}$, we have the three estimates (c is as in (3.1)) 
(i)

$$
\begin{gathered}
c \exp \left(\left(\mu \bar{m}+\frac{\mu(\bar{\sigma})^{2}}{2}\right)(T-t)\right) \leq E^{t, \pi}\left[\exp \left(\mu D\left(h, X_{\tau_{1}}-X_{t}\right)+\mu g\left(\tau_{1}, \pi_{1}\right)\right) 1_{\left\{\tau_{1} \leq T\right\}}\right] \\
\leq c \exp \left(\left(\mu \underline{m}+\frac{(\mu \bar{\sigma})^{2}}{2}\right)(T-t)\right),
\end{gathered}
$$

(ii)

$$
\begin{gathered}
(1-c) \exp \left(\left(\mu \bar{m}+\frac{\mu(\bar{\sigma})^{2}}{2}\right)(T-t)\right) \leq E^{t, \pi}\left[\exp \left(\mu D\left(h, X_{T}-X_{t}\right)\right) 1_{\left\{\tau_{1}>T\right\}}\right] \\
\leq(1-c) \exp \left(\left(\mu \underline{m}+\frac{(\mu \bar{\sigma})^{2}}{2}\right)(T-t)\right)
\end{gathered}
$$

and

(iii)

$$
\left(\underline{m}+\frac{\mu \bar{\sigma}^{2}}{2}\right)(T-t) \leq \hat{\xi}(t, \pi, h ; g) \leq\left(\bar{m}+\frac{\bar{\sigma}^{2}}{2}\right)(T-t) .
$$

Furthermore, for all $g \in \mathcal{G}$, the function $\exp (\mu \hat{\xi}(t, \pi, h ; g))$ is continuous with respect to $h$ and for each $g \in \mathcal{G}_{1}$ we have

$$
\begin{aligned}
& \mid \exp (\mu \hat{\xi}(t, \pi, h ; g)-\exp (\mu \hat{\xi}(t, \bar{\pi}, h ; g)) \mid \\
\leq & \frac{2}{\log 3} d_{H}(\pi, \bar{\pi}) \exp \left(\left(\mu \underline{m}+\frac{(\mu \bar{\sigma})^{2}}{2}\right)(T-t)\right)(1+c),
\end{aligned}
$$

while for each $g \in \mathcal{G}_{2}$ we have

$$
\begin{aligned}
& \mid \exp (\mu \hat{\xi}(t, \pi, h ; g)-\exp (\mu \hat{\xi}(\bar{t}, \pi, h ; g)) \mid \\
\leq & \exp \left(\left(\mu \underline{m}+\frac{(\mu \bar{\sigma})^{2}}{2}\right)(T-t)\right)\left(2 \underline{\underline{n}}\left(e^{\underline{n}(t-\bar{t})}-1\right)+l(t-\bar{t})+c k(t-\bar{t})\right) .
\end{aligned}
$$

Proof. See the Appendix

\subsection{Basic approximation results}

This section is intended to prepare for one of the two main results in Theorem 4.1 below, namely the approximation of the optimal value function, which involves a kind of "value iteration". We start by giving two definitions.

Definition 3.4. Defining the operator $J_{\mu}$ on $C\left([0, T] \times \mathcal{S}_{N}\right)$ by

$$
J_{\mu} g(t, \pi):=\sup _{h \in \bar{H}_{m}} \hat{\xi}(t, \pi, h ; g)
$$

let, for $g \in \mathcal{G}$,

$$
J_{\mu}^{0} g(t, \pi):=g(t, \pi)
$$

and, for $n \geq 1$,

$$
J_{\mu}^{n} g(t, \pi):=J_{\mu}\left(J_{\mu}^{n-1} g(t, \pi)\right)
$$


Definition 3.5. Put

$$
\bar{W}^{0}(t, \pi):=\sup _{h \in \bar{H}_{m}} \bar{W}^{0}(t, \pi, h)=\sup _{h \in \bar{H}_{m}} \frac{1}{\mu} \log E\left[e^{\mu D\left(h, X_{T}-X_{t}\right)}\right]
$$

and let ("value iteration")

$$
\bar{W}^{n}(t, \pi):=J_{\mu}^{n} \bar{W}^{0}(t, \pi)
$$

Then, we have the following proposition as a direct consequence of Proposition 3.1.

Proposition 3.3. For $\bar{W}^{0}(t, \pi)$ defined above we have that $\bar{W}^{0}(t, \pi) \in \mathcal{G}_{1} \cap \mathcal{G}_{2}$ by setting $k(t)=$ $l(t)$.

Proof. By (3.11) in Proposition 3.1 we see that $\bar{W}^{0}(t, \pi) \in \mathcal{G}$ and, by (3.12) and (3.13) in Proposition 3.1 we also see that it belongs to $\mathcal{G}_{1} \cap \mathcal{G}_{2}$ with $k(t)=l(t)$.

We have now

Lemma 3.2. For $g \in \mathcal{G}_{1} \cap \mathcal{G}_{2}$ one has that $J_{\mu} g(t, \pi)$ is continuous with respect to $t, \pi$ and $J_{\mu} g \in \mathcal{G}$.

Proof. For $a, b \geq \gamma>0,|a-b|<\varepsilon, \varepsilon>0$, one has

$$
\begin{aligned}
& \log a-\log b=\log (a / b)=\log (1+(a-b) / b) \leq \epsilon / \gamma \\
& \log b-\log a=\log (b / a)=\log (1+(b-a) / a) \leq \epsilon / \gamma \\
& \Rightarrow|\log a-\log b| \leq \epsilon / \gamma,
\end{aligned}
$$

where we have used the inequality $\log (1+x) \leq x$ for $x \geq 0$. We set $a=\exp (\mu \hat{\xi}(t, \pi, h ; g))$, $b=\exp (\mu \hat{\xi}(t, \bar{\pi}, h ; g))$ and use Proposition 3.2(iii), setting $\gamma=\exp \left(\left\{\mu \bar{m}+\frac{\mu \bar{\sigma}^{2}}{2}\right\}(T-t)\right)$. From (3.21) in Proposition 3.2 it then follows that

$$
\begin{aligned}
& |\hat{\xi}(t, \pi, h ; g)-\hat{\xi}(t, \bar{\pi}, h ; g)| \\
\leq & \frac{1}{|\mu|} \frac{2}{\log 3} d_{H}(\pi, \bar{\pi}) \exp \left(\left(\mu(\underline{m}-\bar{m})+\frac{\mu(\mu-1)(\bar{\sigma})^{2}}{2}\right)(T-t)\right)(1+c) \\
\leq & \frac{1}{|\mu|} \frac{2}{\log 3} d_{H}(\pi, \bar{\pi}) \exp \left(\left(\mu(\underline{m}-\bar{m})+\frac{\mu(\mu-1)(\bar{\sigma})^{2}}{2}\right) T\right)(1+c) .
\end{aligned}
$$

On the other hand, by analogous reasoning, from (3.22) in Proposition 3.2 it follows that

$$
\begin{aligned}
|\hat{\xi}(t, \pi, h ; g)-\hat{\xi}(\bar{t}, \pi, h ; g)| \leq & \frac{1}{|\mu|} \exp \left(\left(\mu(\underline{m}-\bar{m})+\frac{\mu(\mu-1)(\bar{\sigma})^{2}}{2}\right)(T-t)\right) \\
& \times\left(2\left(\frac{\bar{n}}{\underline{n}}\right)\left(e^{\underline{n}(t-\bar{t})}-1\right)+l(t-\bar{t})+c k(t-\bar{t})\right) \\
\leq & \frac{1}{|\mu|} \exp \left(\left(\mu(\underline{m}-\bar{m})+\frac{\mu(\mu-1)(\bar{\sigma})^{2}}{2}\right) T\right) \\
& \times\left(2\left(\frac{\bar{n}}{\underline{n}}\right)\left(e^{\underline{n}(t-\bar{t})}-1\right)+l(t-\bar{t})+c k(t-\bar{t})\right),
\end{aligned}
$$

namely $\hat{\xi}(t, \pi, h ; g)$ is continuous with respect to $(t, \pi)$, uniformly with respect to $h$, and hence $J_{\mu} g(t, \pi)$ is continuous. Further, because of Proposition 3.2 (iii) we see that $J_{\mu} g \in \mathcal{G}$. 
Corollary 3.1. Under the assumptions of Lemma 3.2, $J_{\mu}^{n} g \in \mathcal{G}$ for $n \geq 0$. Furthermore, there exists a Borel function $\hat{h}^{(n)}(t, \pi)$ such that

$$
\sup _{h \in \bar{H}_{m}} \hat{\xi}\left(t, \pi, h ; J_{\mu}^{n} g\right)=\hat{\xi}\left(t, \pi, \hat{h}^{(n)}(t, \pi) ; J_{\mu}^{n} g\right), \quad n \geq 0 .
$$

Proof. Similar arguments to the proof of Lemma 3.2 apply to see that $J_{\mu}^{n} g \in \mathcal{G}$. Moreover, since $\bar{H}_{m}$ is compact and $\hat{\xi}(t, \pi, h ; g)$ is a bounded continuous function on $[0, T] \times \mathcal{S}_{N} \times \bar{H}_{m}$, there exists a Borel function $\hat{h}^{(0)}(t, \pi)$ such that $\sup _{h \in \bar{H}_{m}} \hat{\xi}(t, \pi, h ; g)=\hat{\xi}\left(t, \pi, \hat{h}^{(0)}(t, \pi) ; g\right)$. By the same reasoning we have (3.31) for general $n \geq 1$.

In what follows we denote by $\|g\|$ the norm of a function $g \in C\left([0, T] \times \mathcal{S}_{N}\right)$, namely

$$
\|g\|:=\sup _{(t, \pi) \in[0, T] \times \mathcal{S}_{N}}|g(t, \pi)| .
$$

Lemma 3.3. For each $g \in \mathcal{G}$ and $n \geq 1$, we have the following estimate

$$
\begin{aligned}
& \left\|J_{\mu}^{n+1} g(t, \pi)-J_{\mu}^{n} g(t, \pi)\right\| \\
\leq & \frac{c^{n}}{|\mu|} \exp \left(\left\{\mu(\underline{m}-\bar{m})+\frac{\mu(\mu-1) \bar{\sigma}^{2}}{2}\right\} T\right)\left|1-\exp \left(\mu\left\|J_{\mu} g(t, \pi)-g(t, \pi)\right\|\right)\right|
\end{aligned}
$$

where, recall (3.1), $c \in(0,1)$.

Proof. Let us first prove that, for $n \geq 1$,

$$
\left|\exp \left\{\mu J_{\mu}^{n+1} g(t, \pi)\right\}-\exp \left\{\mu J_{\mu}^{n} g(t, \pi)\right\}\right| \leq c^{n} e^{\left(\mu \underline{m}+\frac{(\mu \bar{\sigma})^{2}}{2}\right)(T-t)}\left|1-e^{\mu\left\|J_{\mu} g-g\right\|}\right| .
$$

To prove it for $n=1$, using Proposition 3.2 (i), we see that

$$
\begin{aligned}
& \left|\exp \left(\mu \hat{\xi}\left(t, \pi, h ; J_{\mu} g\right)\right)-\exp (\mu \hat{\xi}(t, \pi, h ; g))\right| \\
\leq & E^{t, \pi}\left[e^{\mu D\left(h, X_{\tau_{1}}-X_{t}\right)}\left|e^{\mu J_{\mu} g\left(\tau_{1}, \pi_{1}\right)}-e^{\mu g\left(\tau_{1}, \pi_{1}\right)}\right| 1_{\left\{\tau_{1} \leq T\right\}}\right] \\
= & E^{t, \pi}\left[e^{\mu D\left(h, X_{\tau_{1}}-X_{t}\right)+\mu J_{\mu} g\left(\tau_{1}, \pi_{1}\right)}\left|1-e^{\mu\left(g\left(\tau_{1}, \pi_{1}\right)-J_{\mu} g\left(\tau_{1}, \pi_{1}\right)\right)}\right| 1_{\left\{\tau_{1} \leq T\right\}}\right] \\
\leq & \left|1-e^{\mu\left\|J_{\mu} g-g\right\|}\right| E^{t, \pi}\left[e^{\mu D\left(h, X_{\tau_{1}}-X_{t}\right)+\mu J_{\mu} g\left(\tau_{1}, \pi_{1}\right)} 1_{\left\{\tau_{1} \leq T\right\}}\right] \\
\leq & c e^{\mu \underline{m}(T-t)+\frac{(\mu \bar{\sigma})^{2}}{2}(T-t)}\left|1-e^{\mu\left\|J_{\mu} g-g\right\|}\right| .
\end{aligned}
$$

Then, we have

$$
\begin{aligned}
\left|e^{\mu J_{\mu}^{2} g(t, \pi)}-e^{\mu J_{\mu} g(t, \pi)}\right| & =\left|e^{\mu \sup _{h} \hat{\xi}\left(t, \pi, h ; J_{\mu} g\right)}-e^{\mu \sup _{h} \hat{\xi}(t, \pi, h ; g)}\right| \\
& =\left|\inf _{h} e^{\mu \hat{\xi}\left(t, \pi, h ; J_{\mu} g\right)}-\inf _{h} e^{\mu \hat{\xi}(t, \pi, h ; g)}\right| \\
& \leq \sup _{h}\left|e^{\mu \hat{\xi}\left(t, \pi, h ; J_{\mu} g\right)}-e^{\mu \hat{\xi}(t, \pi, h ; g)}\right| \\
& \leq c e^{\mu \underline{m}(T-t)+\frac{(\mu \bar{\sigma} 2)}{2}(T-t)}\left|1-e^{\mu\left\|J_{\mu} g-g\right\|}\right| .
\end{aligned}
$$


Assuming that (3.34) holds for $n-1$, we will prove it for $n$. Using again Proposition 3.2 (i)

$$
\begin{aligned}
& \left|e^{\mu J_{\mu}^{n+1} g(t, \pi)}-e^{\mu J_{\mu}^{n} g(t, \pi)}\right|=\left|e^{\mu \sup _{h} \hat{\xi}\left(t, \pi, h ; J_{\mu}^{n} g\right)}-e^{\mu \sup _{h} \hat{\xi}\left(t, \pi, h ; J_{\mu}^{n-1} g\right)}\right| \\
& \quad=\left|\inf _{h} e^{\mu \hat{\xi}\left(t, \pi, h ; J_{\mu}^{n} g\right)}-\inf _{h} e^{\mu \hat{\xi}\left(t, \pi, h ; J_{\mu}^{n-1} g\right)}\right| \\
& \quad \leq \sup _{h}\left|e^{\mu \hat{\xi}\left(t, \pi, h ; J_{\mu}^{n} g\right)}-e^{\mu \hat{\xi}\left(t, \pi, h ; J_{\mu}^{n-1} g\right)}\right| \\
& \quad \leq \sup _{h} E^{t, \pi}\left[e^{\mu D\left(h, X_{\tau_{1}}-X_{t}\right)}\left|e^{\mu J_{\mu}^{n} g\left(\tau_{1}, \pi_{1}\right)}-e^{\mu J_{\mu}^{n-1} g\left(\tau_{1}, \pi_{1}\right)}\right| 1_{\left\{\tau_{1} \leq T\right\}}\right] \\
& \quad \leq \sup _{h} E^{t, \pi}\left[e^{\mu D\left(h, X_{\tau_{1}}-X_{t}\right)} c^{n-1} e^{\left(\mu \underline{m}+\frac{(\mu \bar{\sigma})^{2}}{2}\right)\left(T-\tau_{1}\right)} 1_{\left\{\tau_{1} \leq T\right\}}\left|1-e^{\mu\left\|J_{\mu} g-g\right\|}\right|\right] \\
& \quad \leq c^{n} e^{\left(\mu \underline{m}+\frac{(\mu \bar{\sigma})^{2}}{2}\right)(T-t)}\left|1-e^{\mu\left\|J_{\mu} g-g\right\|}\right| .
\end{aligned}
$$

Thus, (3.34) has been proved. Now we can complete the proof. Indeed, by using (3.28), we have

$$
\begin{aligned}
& \left|J_{\mu}^{n+1} g(t, \pi)-J_{\mu}^{n} g(t, \pi)\right|=\frac{1}{|\mu|}\left|\mu J_{\mu}^{n+1} g(t, \pi)-\mu J_{\mu}^{n} g(t, \pi)\right| \\
& \quad \leq \frac{1}{|\mu|} e^{-\mu \bar{m}(T-t)-\frac{\mu \bar{\sigma}^{2}}{2}(T-t)}\left|e^{\mu J_{\mu}^{n+1} g(t, \pi)}-e^{\mu J_{\mu}^{n} g(t, \pi)}\right| \\
& \quad \leq \frac{c^{n}}{|\mu|} e^{\mu(\underline{m}-\bar{m})(T-t)+\frac{\left(\mu^{2}-\mu\right) \bar{\sigma}^{2}}{2}(T-t)}\left|1-e^{\mu\left\|J_{\mu} g-g\right\|}\right| \\
& \quad \leq \frac{c^{n}}{|\mu|} e^{\mu(\underline{m}-\bar{m}) T+\frac{\left(\mu^{2}-\mu\right) \bar{\sigma}^{2}}{2} T}\left|1-e^{\mu\left\|J_{\mu} g-g\right\|}\right|
\end{aligned}
$$

and hence obtain the present lemma by taking supremum with respect to $(t, \pi)$.

Corollary 3.2. For $g \in \mathcal{G}_{1} \cap \mathcal{G}_{2}$ we have that $\left\{J_{\mu}^{n} g(t, \pi)\right\}$ is a Cauchy sequence in $\mathcal{G}$ and, therefore, $\exists \lim _{n \rightarrow \infty} J_{\mu}^{n} g(t, \pi) \in \mathcal{G}$.

Furthermore, for each $g_{1}, g_{2} \in \mathcal{G}$, we have the estimates:

$$
\begin{aligned}
& \left\|J_{\mu} g_{1}-J_{\mu} g_{2}\right\| \\
\leq & \frac{c}{|\mu|} \exp \left(\left\{\mu(\underline{m}-\bar{m})+\frac{\mu(\mu-1) \bar{\sigma}^{2}}{2}\right\} T\right)\left|1-\exp \left(\mu\left\|g_{1}-g_{2}\right\|\right)\right| \\
\leq & c \exp \left(\left\{\mu(\underline{m}-\bar{m})+\frac{\mu(\mu-1) \bar{\sigma}^{2}}{2}\right\} T\right)\left\|g_{1}-g_{2}\right\| .
\end{aligned}
$$

Proof. By Corollary 3.1, $\left\{J_{\mu}^{n} g(t, \pi)\right\} \subset \mathcal{G}$. Further, by using Lemma 3.3, we can see that $\left\{J_{\mu}^{n} g(t, \pi)\right\}$ is a Cauchy sequence in $\mathcal{G}$. The proof of (3.38) is similar to that of Lemma 3.3.

Proposition 3.4. We have that $\left\{\bar{W}^{n}(t, \pi)\right\}$ given by (3.27) is a Cauchy sequence in $\mathcal{G}$ and therefore $\exists \lim _{n \rightarrow \infty} \bar{W}^{n}(t, \pi) \in \mathcal{G}$.

Proof. Thanks to Proposition 3.3, $\bar{W}^{0}(t, \pi)$ belongs to $\mathcal{G}_{1} \cap \mathcal{G}_{2}$ with $k(t)=l(t)$ and we see that $\left\{\bar{W}^{n}(t, \pi)\right\}$ is a Cauchy sequence in $\mathcal{G}$ by Corollary 3.2 .

\subsection{Limiting results}

In this subsection we perform some passages to the limit, which will complete our preliminary analysis in view of the main result in the next section.

We start by defining some relevant quantities. 
Definition 3.6. We set

$$
\bar{W}(t, \pi):=\lim _{n \rightarrow \infty} \bar{W}^{n}(t, \pi),
$$

which is justified by the previous Proposition 3.4,

$$
W(t, \pi):=\sup _{h \in \mathcal{A}} W(t, \pi, h .),
$$

(this definition was already given in (2.36), but we iterate it here for convenience in the present context), and

$$
W^{n}(t, \pi):=\sup _{h \in \mathcal{A}^{n}} W(t, \pi, h .)
$$

where $W(t, \pi, h$.$) is the criterion function defined in (2.35).$

The next lemma particularizes the representation result of Lemma 3.1.

Lemma 3.4. For all $n \geq 0$ and $h \in \mathcal{A}^{n}$, we have the following equation

$$
\begin{aligned}
W(t, \pi, h .)= & \frac{1}{\mu} \log E^{t, \pi}\left[\operatorname { e x p } \left(\mu \sum_{k=1}^{n} D\left(h_{k-1}, X_{T \wedge \tau_{k}}-X_{\tau_{k-1}}\right) 1_{\left\{\tau_{k-1}<T\right\}}\right.\right. \\
& \left.\left.+\mu D\left(h_{n}, X_{T}-X_{\tau_{n}}\right) 1_{\left\{\tau_{n}<T\right\}}\right)\right] \\
= & \frac{1}{\mu} \log E^{t, \pi}\left[\operatorname { e x p } \left(\mu \sum_{k=1}^{n} D\left(h_{k-1}, X_{T \wedge \tau_{k}}-X_{\tau_{k-1}}\right) 1_{\left\{\tau_{k-1}<T\right\}}\right.\right. \\
& \left.\left.+\mu \bar{W}^{0}\left(\tau_{n}, \pi_{n}, h_{n}\right) 1_{\left\{\tau_{n} \leq T\right\}}\right)\right] .
\end{aligned}
$$

Proof. By Lemma 3.1 and the definition of $\bar{W}^{0}(t, \pi, h)$ it suffices to prove the following equation for all $n>0, k \geq n+1, h \in \mathcal{A}^{n}$. For $\tau_{k}<T \leq \tau_{k+1}$

$$
\begin{aligned}
& \exp \left(\mu \sum_{i=n}^{k-1} D\left(h_{i}, X_{\tau_{i+1}}-X_{\tau_{i}}\right)+\mu D\left(h_{k}, X_{T}-X_{\tau_{k}}\right)\right) \\
= & \exp \left(\mu D\left(h_{n}, X_{T}-X_{\tau_{n}}\right)\right) .
\end{aligned}
$$

It can be seen as follows.

$$
\begin{aligned}
& \exp \left(\mu \sum_{i=n}^{k-1} D\left(h_{i}, X_{\tau_{i+1}}-X_{\tau_{i}}\right)+\mu D\left(h_{k}, X_{T}-X_{\tau_{k}}\right)\right) \\
= & \prod_{i=n}^{k-1}\left(\sum_{j=0}^{m} h_{i}^{j} \frac{S_{i+1}^{j}}{S_{i}^{j}}\right)^{\mu}\left(\sum_{j=0}^{m} h_{k}^{j} \frac{S_{T}^{j}}{S_{k}^{j}}\right)^{\mu}=\prod_{i=n}^{k-1}\left(\sum_{j=0}^{m} \frac{N_{i}^{j} S_{i+1}^{j}}{V_{i}}\right)^{\mu}\left(\sum_{j=0}^{m} \frac{N_{k}^{j} S_{T}^{j}}{V_{k}}\right)^{\mu} \\
= & \prod_{i=n}^{k-1}\left(\sum_{j=0}^{m} \frac{N_{i+1}^{j} S_{i+1}^{j}}{V_{i}}\right)^{\mu}\left(\sum_{j=0}^{m} \frac{N_{k}^{j} S_{T}^{j}}{V_{k}}\right)^{\mu}=\prod_{i=n}^{k-1}\left(\frac{V_{i+1}}{V_{i}}\right)^{\mu}\left(\sum_{j=0}^{m} \frac{N_{T}^{j} S_{T}^{j}}{V_{k}}\right)^{\mu} \\
= & \left(\frac{N_{T} S_{T}}{V_{n}}\right)^{\mu}=\left(\frac{V_{T}}{V_{n}}\right)^{\mu}=\exp \left(\mu D\left(h_{n}, X_{T}-X_{\tau_{n}}\right)\right),
\end{aligned}
$$

where we have used (3.5), the definition of $\mathcal{A}^{n}$ and the self financing property of the investment strategy. 
Corollary 3.3. We have the following equation

$$
\begin{aligned}
W^{n}(t, \pi)= & \sup _{h \in \mathcal{A}^{n}} \frac{1}{\mu} \log E^{t, \pi}\left[\operatorname { e x p } \left(\mu \sum_{k=1}^{n} D\left(h_{k-1}, X_{T \wedge \tau_{k}}-X_{\tau_{k-1}}\right) 1_{\left\{\tau_{k-1}<T\right\}}\right.\right. \\
& \left.\left.+\mu \bar{W}^{0}\left(\tau_{n}, \pi_{n}, h_{n}\right) 1_{\left\{\tau_{n} \leq T\right\}}\right)\right],
\end{aligned}
$$

for $n \geq 0, t \in[0, T], \pi \in \mathcal{S}_{N}$.

Proposition 3.5. For each $n \geq 0$, we have

$$
\bar{W}^{n}(t, \pi)=W^{n}(t, \pi) .
$$

Furthermore,

$$
\bar{W}(t, \pi)=W(t, \pi)
$$

Proof. See the Appendix.

The next proposition is in the spirit of a Dynamic Programming principle, namely

Proposition 3.6. We have $W=J_{\mu} W$. Namely, $W(t, \pi)$ satisfies the following equation

$$
\begin{aligned}
& W(t, \pi) \\
& =\sup _{h \in \bar{H}_{m}} \frac{1}{\mu} \log E^{t, \pi}\left[\exp \left(\mu D\left(h, X_{T \wedge \tau_{1}}-X_{t}\right)+\mu W\left(\tau_{1}, \pi_{1}\right) 1_{\left\{\tau_{1} \leq T\right\}}\right)\right] .
\end{aligned}
$$

Proof. We have, by using (3.38),

$$
\begin{aligned}
\left\|W-J_{\mu} W\right\| & \leq\left\|W-J_{\mu} \bar{W}^{n}\right\|+\left\|J_{\mu} \bar{W}^{n}-J_{\mu} W\right\| \\
& \leq\left\|W-\bar{W}^{n+1}\right\|+C_{1}\left\|\bar{W}^{n}-W\right\|,
\end{aligned}
$$

where $C_{1}=c \exp \left(\left\{\mu(\underline{m}-\bar{m})+\frac{\mu(\mu-1) \bar{\sigma}^{2}}{2}\right\} T\right)$. Hence, by sending $n$ to $\infty$, we see that $\left\|W-J_{\mu} W\right\|=$ 0 .

\section{Main Theorem}

From the preliminary analysis in Section 3 we obtain now the main result of this paper, namely an approximation result and a Dynamic Programming-type principle for the power-utility maximization problem.

\section{Theorem 4.1.}

(i) Approximation theorem

$\bar{W}^{n}$ computed according to (3.27) in Definition 3.5 are approximations to the solution of the original problem in the sense that, for any $\epsilon>0, n>n_{\epsilon}$,

$$
\left\|W-\bar{W}^{n}\right\|<\epsilon,
$$

where,

$$
n_{\epsilon}:=\frac{\log (1-c)|\mu|+\log \epsilon-\log \left|1-\exp \left(\mu\left\|J_{\mu}^{1} \bar{W}^{0}-J_{\mu}^{0} \bar{W}^{0}\right\|\right)\right|-\left\{\mu(\underline{m}-\bar{m})+\frac{\mu(\mu-1) \bar{\sigma}^{2}}{2}\right\} T}{\log c} .
$$


(ii) Dynamic programming principle:

for $n \geq 0$

$$
\begin{aligned}
& W(t, \pi) \\
& =\sup _{h \in \mathcal{A}^{n}} \frac{1}{\mu} \log E^{t, \pi}\left[\operatorname { e x p } \left(\mu \sum_{k=1}^{n} D\left(h_{k-1}, X_{T \wedge \tau_{k}}-X_{\tau_{k-1}}\right) 1_{\left\{\tau_{k-1}<T\right\}}\right.\right. \\
& \left.\left.+\mu W\left(\tau_{n}, \pi_{n}\right) 1_{\left\{\tau_{n} \leq T\right\}}\right)\right] .
\end{aligned}
$$

(iii) Optimal value and optimal strategy for the Power Utility Maximization Problem For the utility maximization under initial condition $V_{0}=v_{0}, \tau_{0}=0, \pi_{0}=\pi$ we have

$$
\begin{aligned}
\sup _{h \in \mathcal{A}} & \frac{1}{\mu} \log E^{0, \pi}\left[V_{T}^{\mu}\right]=\log v_{0} \\
& \quad+\frac{1}{\mu} \log E^{0, \pi}\left[\exp \left(\mu \sum_{k=1}^{\infty} D\left(\hat{h}_{k-1}, X_{T \wedge \tau_{k}}-X_{\tau_{k-1}}\right) 1_{\left\{\tau_{k-1}<T\right\}}\right)\right],
\end{aligned}
$$

where the optimal strategy in the $k$-th period, namely $\hat{h}_{k}$, is given by

$$
\hat{h}_{k}=\hat{h}\left(\tau_{k}, \pi_{k}\right)
$$

with $\hat{h}(\tau, \pi)$ defined by

$$
\begin{aligned}
& \sup _{h \in \bar{H}_{m}} \frac{1}{\mu} \log E^{t, \pi}\left[\exp \left(\mu D\left(h, X_{T \wedge \tau_{1}}-X_{t}\right)+\mu W\left(\tau_{1}, \pi_{1}\right) 1_{\left\{\tau_{1} \leq T\right\}}\right)\right] \\
= & \frac{1}{\mu} \log E^{t, \pi}\left[\exp \left(\mu D\left(\hat{h}(t, \pi), X_{T \wedge \tau_{1}}-X_{t}\right)+\mu W\left(\tau_{1}, \pi_{1}\right) 1_{\left\{\tau_{1} \leq T\right\}}\right)\right] .
\end{aligned}
$$

Proof. First, we prove $(i)$. For any $n$

$$
\begin{aligned}
& \left\|\bar{W}-\bar{W}^{n}\right\|=\left\|\lim _{k \rightarrow \infty} \bar{W}^{n+k}-\bar{W}^{n}\right\|=\lim _{k \rightarrow \infty}\left\|\bar{W}^{n+k}-\bar{W}^{n}\right\| \\
& \quad \leq \lim _{k \rightarrow \infty} \sum_{i=0}^{k-1}\left\|\bar{W}^{n+i+1}-\bar{W}^{n+i}\right\|=\lim _{k \rightarrow \infty} \sum_{i=0}^{k-1}\left\|J_{\mu}^{n+i+1} \bar{W}^{0}-J_{\mu}^{n+i} \bar{W}^{0}\right\| \\
& \quad \leq \frac{1}{|\mu|}\left|1-\exp \left(\mu\left\|J_{\mu}^{1} \bar{W}^{0}-J_{\mu}^{0} \bar{W}^{0}\right\|\right)\right| \exp \left(\left\{\mu(\underline{m}-\bar{m})+\frac{\mu(\mu-1) \bar{\sigma}^{2}}{2}\right\} T\right) \sum_{i=0}^{\infty} c^{i+n} \\
& \quad=\frac{1}{|\mu|}\left|1-\exp \left(\mu\left\|J_{\mu}^{1} \bar{W}^{0}-J_{\mu}^{0} \bar{W}^{0}\right\|\right)\right| \exp \left(\left\{\mu(\underline{m}-\bar{m})+\frac{\mu(\mu-1) \bar{\sigma}^{2}}{2}\right\} T\right) c^{n} \sum_{i=0}^{\infty} c^{i} \\
& \quad=\frac{c^{n}}{(1-c)|\mu|}\left|1-\exp \left(\mu\left\|J_{\mu}^{1} \bar{W}^{0}-J_{\mu}^{0} \bar{W}^{0}\right\|\right)\right| \exp \left(\left\{\mu(\underline{m}-\bar{m})+\frac{\mu(\mu-1) \bar{\sigma}^{2}}{2}\right\} T\right),
\end{aligned}
$$

where we have used Lemma 3.3.

Next, we prove (ii). Proceeding analogously to the proof of Lemma 5.5 in the Appendix, for $n, m \in \mathbf{N}$, we take a sequence of functions $\tilde{h}^{n, k}(t, \pi), k=0,1, \ldots, n$ such that

$$
\sup _{h \in H^{m}} \hat{\xi}\left(t, \pi, h ; J_{\mu}^{n+m-k-1} \bar{W}^{0}\right)=\hat{\xi}\left(t, \pi, \tilde{h}^{n, k}(t, \pi) ; J_{\mu}^{n+m-k-1} \bar{W}^{0}\right)
$$


and set

$$
\tilde{h}_{k}^{n, k}:=\tilde{h}^{n, k}\left(\tau_{k}, \pi_{k}\right), \quad k=0,1, \ldots, n
$$

and

$$
\tilde{h}_{k}^{n, k}=\gamma\left(\tilde{X}_{\tau_{k}}-\tilde{X}_{\tau_{n}}, \tilde{h}_{n}^{n, n}\right), \quad k \geq n+1 .
$$

Then, $\tilde{h}^{(n)}=\left\{\tilde{h}_{k}^{n, k}\right\}_{k} \in \mathcal{A}^{n}$. Therefore, similarly to the proof of Lemma 5.5, it follows that

$$
\begin{gathered}
\bar{W}^{n+m}=\frac{1}{\mu} \log E^{t, \pi}\left[\operatorname { e x p } \left\{\mu \sum_{k=1}^{n} D\left(\tilde{h}^{n, k-1}\left(\tau_{k-1}, \pi_{k-1}\right), X_{T \wedge \tau_{k}}-X_{\tau_{k-1}}\right) 1_{\left\{\tau_{k-1} \leq T\right\}}\right.\right. \\
\left.\left.+\mu J_{\mu}^{m} \bar{W}^{0}\left(\tau_{n}, \pi_{n}\right) 1_{\left\{\tau_{n} \leq T\right\}}\right\}\right] .
\end{gathered}
$$

Since $J_{\mu}^{m} \bar{W}^{0} \leq W$, we have

$$
\begin{gathered}
\bar{W}^{n+m} \leq \frac{1}{\mu} \log E^{t, \pi}\left[\operatorname { e x p } \left\{\mu \sum_{k=1}^{n} D\left(\tilde{h}^{n, k-1}\left(\tau_{k-1}, \pi_{k-1}\right), X_{T \wedge \tau_{k}}-X_{\tau_{k-1}}\right) 1_{\left\{\tau_{k-1} \leq T\right\}}\right.\right. \\
\left.\left.\quad+\mu W\left(\tau_{n}, \pi_{n}\right) 1_{\left\{\tau_{n} \leq T\right\}}\right\}\right] \\
\leq \sup _{h \in \mathcal{A}^{n}} \frac{1}{\mu} \log E^{t, \pi}\left[\operatorname { e x p } \left\{\mu \sum_{k=1}^{n} D\left(h_{k-1}, X_{T \wedge \tau_{k}}-X_{\tau_{k-1}}\right) 1_{\left\{\tau_{k-1} \leq T\right\}}\right.\right. \\
\left.\left.\quad+\mu W\left(\tau_{n}, \pi_{n}\right) 1_{\left\{\tau_{n} \leq T\right\}}\right\}\right] .
\end{gathered}
$$

Therefore, we obtain

$$
\begin{gathered}
W(t, \pi) \leq \sup _{h \in \mathcal{A}^{n}} \frac{1}{\mu} \log E^{t, \pi}\left[\operatorname { e x p } \left\{\mu \sum_{k=1}^{n} D\left(h_{k-1}, X_{T \wedge \tau_{k}}-X_{\tau_{k-1}}\right) 1_{\left\{\tau_{k-1} \leq T\right\}}\right.\right. \\
\left.\left.+\mu W\left(\tau_{n}, \pi_{n}\right) 1_{\left\{\tau_{n} \leq T\right\}}\right\}\right],
\end{gathered}
$$

by letting $m \rightarrow \infty$. On the other hand, for each $h \in \mathcal{A}^{n}$, it follows that

$$
\begin{gathered}
\bar{W}^{n+m}(t, \pi) \geq \frac{1}{\mu} \log E^{t, \pi}\left[\operatorname { e x p } \left\{\mu \sum_{k=1}^{n} D\left(h_{k-1}, X_{T \wedge \tau_{k}}-X_{\tau_{k-1}}\right) 1_{\left\{\tau_{k-1} \leq T\right\}}\right.\right. \\
\left.\left.+\mu J_{\mu}^{m} \bar{W}^{0}\left(\tau_{n}, \pi_{n}\right) 1_{\left\{\tau_{n} \leq T\right\}}\right\}\right] .
\end{gathered}
$$

Then, by letting $m \rightarrow \infty$, we have

$$
\begin{gathered}
W(t, \pi) \geq \frac{1}{\mu} \log E^{t, \pi}\left[\operatorname { e x p } \left\{\mu \sum_{k=1}^{n} D\left(h_{k-1}, X_{T \wedge \tau_{k}}-X_{\tau_{k-1}}\right) 1_{\left\{\tau_{k-1} \leq T\right\}}\right.\right. \\
\left.\left.+\mu W\left(\tau_{n}, \pi_{n}\right) 1_{\left\{\tau_{n} \leq T\right\}}\right\}\right] .
\end{gathered}
$$

Hence,

$$
\begin{gathered}
W(t, \pi) \geq \sup _{h \in \mathcal{A}^{n}} \frac{1}{\mu} \log E^{t, \pi}\left[\operatorname { e x p } \left\{\mu \sum_{k=1}^{n} D\left(h_{k-1}, X_{T \wedge \tau_{k}}-X_{\tau_{k-1}}\right) 1_{\left\{\tau_{k-1} \leq T\right\}}\right.\right. \\
\left.\left.+\mu W\left(\tau_{n}, \pi_{n}\right) 1_{\left\{\tau_{n} \leq T\right\}}\right\}\right]
\end{gathered}
$$

and thus we obtain $(i i)$.

Part (iii) is an immediate consequence of the previous results in particular of the proof of point (ii) of this same theorem. 


\section{Appendix}

Proof of Proposition 3.1. The proof is contained in the following two lemmas.

Lemma 5.1. For $t \in[0, T], h \in \bar{H}_{m}$, we have the estimate (3.10).

Proof. Since $x^{\mu}$ is convex, Jensen's inequality applies and we obtain

$$
E^{t, \pi}\left[\left(\sum_{i=0}^{m} h^{i} \exp \left(X_{T}^{i}-X_{t}^{i}\right)\right)^{\mu}\right] \leq E^{t, \pi}\left[\sum_{i=0}^{m} h^{i} \exp \left(\mu\left(X_{T}^{i}-X_{t}^{i}\right)\right)\right] .
$$

For each $i$ and $t \in[0, T]$,

$$
\begin{aligned}
& \underline{m}(T-t) \leq \int_{t}^{T} m_{i}\left(\theta_{s}\right) d s \leq \bar{m}(T-t), \\
& \int_{t}^{T} \sigma_{i}^{2}\left(\theta_{s}\right) d s \leq \bar{\sigma}^{2}(T-t) .
\end{aligned}
$$

Thus, we have

$$
\begin{aligned}
E^{t, \pi}\left[\exp \left(\mu\left(X_{T}^{i}-X_{t}^{i}\right)\right)\right] & =E^{t, \pi}\left[\exp \left(\mu \int_{t}^{T} m_{i}\left(\theta_{s}\right) d s+\frac{\mu^{2}}{2} \int_{t}^{T} \sigma_{i}^{2}\left(\theta_{s}\right) d s\right)\right] \\
& \leq \exp \left(\left(\mu \underline{m}+\frac{(\mu \bar{\sigma})^{2}}{2}\right)(T-t)\right)
\end{aligned}
$$

and

$$
E^{t, \pi}\left[\exp \left(\mu\left(X_{T}^{0}-X_{t}^{0}\right)\right)\right]=\exp \left(\mu r_{0}(T-t)\right) \leq \exp \left(\left(\mu \underline{m}+\frac{(\mu \bar{\sigma})^{2}}{2}\right)(T-t)\right) .
$$

Therefore, from (5.1) it follows that

$$
\begin{aligned}
E^{t, \pi}\left[\left(\sum_{i=0}^{m} h^{i} \exp \left(X_{T}^{i}-X_{t}^{i}\right)\right)^{\mu}\right] & \leq \sum_{i=0}^{m} h^{i} \exp \left(\left(\mu \underline{m}+\frac{(\mu \bar{\sigma})^{2}}{2}\right)(T-t)\right) \\
& =\exp \left(\left(\mu \underline{m}+\frac{(\mu \bar{\sigma})^{2}}{2}\right)(T-t)\right) .
\end{aligned}
$$

To obtain the lower estimate, applying Jensen's inequality yields

$$
\left(E^{t, \pi}\left[\sum_{i=0}^{m} h^{i} \exp \left(X_{T}^{i}-X_{t}^{i}\right)\right]\right)^{\mu} \leq E^{t, \pi}\left[\left(\sum_{i=0}^{m} h^{i} \exp \left(X_{T}^{i}-X_{t}^{i}\right)\right)^{\mu}\right] .
$$

Since $x^{\mu}$ is a decreasing function, we have

$$
\begin{aligned}
\left(\sum_{i=0}^{m} h^{i} E^{t, \pi}\left[\exp \left(X_{T}^{i}-X_{t}^{i}\right)\right]\right)^{\mu} & =\left(\sum_{i=0}^{m} h^{i} E^{t, \pi}\left[\exp \left(\int_{t}^{T} m_{i}\left(\theta_{s}\right) d s+\frac{1}{2} \int_{t}^{T} \sigma_{i}^{2}\left(\theta_{s}\right) d s\right)\right]\right)^{\mu} \\
& \geq\left(\sum_{i=0}^{m} h^{i} \exp \left(\left(\bar{m}+\frac{(\bar{\sigma})^{2}}{2}\right)(T-t)\right)\right)^{\mu} \\
& =\exp \left(\left(\mu \bar{m}+\frac{\mu(\bar{\sigma})^{2}}{2}\right)(T-t)\right) .
\end{aligned}
$$


Lemma 5.2. $\bar{W}^{0}(t, \pi, h)$ in Definition 3.1 (see (3.9)) is a continuous function on $[0, T] \times \mathcal{S}_{N} \times$ $\bar{H}_{m}$ and the estimates (3.12), (3.13) hold.

Proof. Let us first prove the continuity of $\bar{W}^{0}(t, \pi, h)$ with respect to $\pi$. Owing to (2.4) and recalling $p_{j i}(t)$ that was defined in $(2.28)$, we have

$$
\begin{aligned}
& \left|\exp \left(\mu \bar{W}^{0}(t, \pi, h)\right)-\exp \left(\mu \bar{W}^{0}(t, \bar{\pi}, h)\right)\right| \\
= & \mid \int_{\mathbb{R}^{m}}\left(h^{0} \exp \left(r_{0}(T-t)\right)+\sum_{i=1}^{m} h^{i} \exp \left(y^{i}\right)\right)^{\mu} \\
& \times \sum_{i j} E\left[\rho_{0, T-t}^{\theta}(y) \mid \theta_{0}=e_{j}, \theta_{T-t}=e_{i}\right] p_{j i}(T-t)\left(\pi^{j}-\bar{\pi}^{j}\right) d y d s \mid \\
= & \left|\sum_{j} \exp \left(\mu \bar{W}^{0}\left(t, e_{j}, h\right)\right)\left(\pi^{j}-\bar{\pi}^{j}\right)\right| \\
\leq & \exp \left(\left(\mu \underline{m}+\frac{(\mu \bar{\sigma})^{2}}{2}\right)(T-t)\right) \sum_{j}\left|\pi^{j}-\bar{\pi}^{j}\right| \\
\leq & \exp \left(\left(\mu \underline{m}+\frac{(\mu \bar{\sigma})^{2}}{2}\right)(T-t)\right)\left\|\pi^{j}-\bar{\pi}^{j}\right\|_{T V} \\
\leq & \exp \left(\left(\mu \underline{m}+\frac{(\mu \bar{\sigma})^{2}}{2}\right)(T-t)\right) \frac{2}{\log 3} d_{H}(\pi, \bar{\pi}) .
\end{aligned}
$$

Next, we show the continuity of $\bar{W}^{0}(t, \pi, h)$ with respect to $t$. First notice that, due to the time homogeneity of the process $\left(X_{t}, \theta_{t}\right)$,

$$
\begin{aligned}
\exp \left(\mu \bar{W}^{0}(t, \pi, h)\right) & =E^{t, \pi}\left[\exp \left(\mu D\left(h, X_{T}-X_{t}\right)\right)\right] \\
& =E^{0, \pi}\left[\exp \left(\mu D\left(h, X_{T-t}-X_{0}\right)\right)\right] .
\end{aligned}
$$

Notice furthermore that

$$
|D(h, x)-D(h, y)| \leq|x-y|
$$

holds because

$$
\left|\nabla_{x} D(h, x)\right| \leq 1 .
$$

Therefore,

$$
\begin{aligned}
& \left|\exp \left(\mu \bar{W}^{0}(t, \pi, h)\right)-\exp \left(\mu \bar{W}^{0}(\bar{t}, \pi, h)\right)\right| \\
= & \left|E^{0, \pi}\left[\exp \left(\mu D\left(h, X_{T-t}-X_{0}\right)\right)-\exp \left(\mu D\left(h, X_{T-\bar{t}}-X_{0}\right)\right)\right]\right| \\
= & \mid E^{0, \pi}\left[\exp \left(\mu D\left(h, X_{T-t}-X_{0}\right)\right)\left(1-\exp \left(\mu\left(D\left(h, X_{T-\bar{t}}-X_{0}\right)-D\left(h, X_{T-t}-X_{0}\right)\right)\right)\right)\right] \\
\leq & E^{0, \pi}\left[\exp \left(\mu D\left(h, X_{T-t}-X_{0}\right)\right)\left|1-\exp \left(\mu\left|X_{T-\bar{t}}-X_{T-t}\right|\right)\right|\right] \\
= & E^{0, \pi}\left[\exp \left(\mu D\left(h, X_{T-t}-X_{0}\right)\right) E\left[\left|1-\exp \left(\mu\left|X_{T-\bar{t}}-X_{T-t}\right|\right)\right| \mid X_{T-t}\right]\right] \\
= & E^{0, \pi}\left[\exp \left(\mu D\left(h, X_{T-t}-X_{0}\right)\right)\right] l(t-\bar{t}) \\
\leq & \exp \left(\left(\mu \underline{m}+\frac{(\mu \bar{\sigma})^{2}}{2}\right)(T-t)\right) l(t-\bar{t}) .
\end{aligned}
$$


Finally, we prove the continuity with respect to $h$. By the definition of $D(h, x)$ and Jensen's inequality,

$$
\exp (\mu D(h, x))=\left(\sum_{i=0}^{m} h^{i} \exp \left(x^{i}\right)\right)^{\mu} \leq \sum_{i=0}^{m} h^{i} \exp \left(\mu x^{i}\right) \leq \sum_{i=0}^{m} \exp \left(\mu x^{i}\right) .
$$

Therefore, for $m \geq 1$

$$
\exp \left(\mu D\left(h, X_{T}-X_{t}\right)\right) \leq \sum_{i=0}^{m} \exp \left(\mu\left(X_{T}^{i}-X_{t}^{i}\right)\right)
$$

Since $X_{T}-X_{t}=\left\{X_{T}^{i}-X_{t}^{i}\right\}_{i=1, \ldots, m}$ is, conditionally on $\mathcal{F}^{\theta}$, Gaussian with mean $\left\{\int_{t}^{T} m_{i}\left(\theta_{s}\right) d s\right\}_{i=1, \ldots, m}$ and covariance $\left\{\int_{t}^{T}\left(\sigma \sigma^{*}\right)^{i j}\left(\theta_{s}\right) d s\right\}_{i, j=1, \ldots, m}$ we have

$$
E^{t, \pi}\left[\exp \left(\mu\left(X_{T}^{i}-X_{t}^{i}\right)\right)\right]<\infty .
$$

Then, applying the dominated convergence theorem, for $h_{j} \subset \bar{H}_{m}$, s.t. $\lim _{j \rightarrow \infty} h_{j}=h \in \bar{H}_{m}$

$$
\begin{aligned}
\lim _{j \rightarrow \infty} \bar{W}^{0}\left(t, \pi, h_{j}\right) & =\frac{1}{\mu} \log E^{t, \pi}\left[\lim _{j \rightarrow \infty} \exp \left(\mu D\left(h_{j}, X_{T}-X_{t}\right)\right)\right] \\
& =\frac{1}{\mu} \log E^{t, \pi}\left[\exp \left(\mu D\left(h, X_{T}-X_{t}\right)\right)\right] \\
& =\bar{W}^{0}(t, \pi, h) .
\end{aligned}
$$

Proof of Proposition 3.2. Again, the proof is contained in the following two lemmas.

Lemma 5.3. For each $g \in \mathcal{G}$, we have the three estimates (3.18), (3.19) and (3.20).

Proof. Let us first set

$$
I_{1}=E^{t, \pi}\left[\exp \left(\mu D\left(h, X_{\tau_{1}}-X_{t}\right)+\mu g\left(\tau_{1}, \pi_{1}\right)\right) 1_{\left\{\tau_{1} \leq T\right\}}\right]
$$

and

$$
I_{2}=E^{t, \pi}\left[\exp \left(\mu D\left(h, X_{T}-X_{t}\right)\right) 1_{\left\{\tau_{1}>T\right\}}\right] .
$$

Recall also that $n\left(\theta_{t}\right)$ is the intensity of the Cox process describing the observations and that the dynamics of the filter process $\pi_{t}$ was given in Corollary 2.2 in terms of the function $M(t, x, \pi)$. (i) (estimate (3.18)). Since $g \in \mathcal{G}$, from the definition of $\rho_{t, T}^{\theta}(z)$ in (2.12) and from (5.2) we 
obtain

$$
\begin{aligned}
& I_{1}= E^{t, \pi}\left[\int_{t}^{T} \int_{\mathbb{R}^{m}}\left(h^{0} \exp \left(r_{0}(s-t)\right)+\sum_{i=1}^{m} h^{i} \exp \left(z^{i}\right)\right)^{\mu} \exp (\mu g(s, M(s-t, z, \pi)))\right. \\
&\left.\times \rho_{t, s}^{\theta}(z) n\left(\theta_{s}\right) \exp \left(-\int_{t}^{s} n\left(\theta_{u}\right) d u\right) d s d z\right] \\
& \geq \quad E^{t, \pi}\left[\int_{t}^{T} \int_{\mathbb{R}^{m}}\left(h^{0} \exp \left(r_{0}(s-t)\right)+\sum_{i=1}^{m} h^{i} \exp \left(z^{i}\right)\right)^{\mu} \rho_{t, s}^{\theta}(z) d z\right. \\
&\left.\times \exp \left(\left(\mu \bar{m}+\frac{\mu \bar{\sigma}^{2}}{2}\right)(T-s)\right) n\left(\theta_{s}\right) \exp \left(-\int_{t}^{s} n\left(\theta_{u}\right) d u\right) d s\right] \\
& \geq \quad E^{t, \pi}\left[\int_{t}^{T}\left(h^{0} \exp \left(r_{0}(s-t)\right)+\sum_{i=1}^{m} h^{i} \int_{\mathbb{R}^{m}} \exp \left(z^{i}\right) \rho_{t, s}^{\theta}(z) d z\right)^{\mu}\right. \\
& \quad \\
&\left.\quad \quad \quad \exp \left(\left(\mu \bar{m}+\frac{\mu \bar{\sigma}^{2}}{2}\right)(T-s)\right) n\left(\theta_{s}\right) \exp \left(-\int_{t}^{s} n\left(\theta_{u}\right) d u\right) d s\right] \\
& \quad E^{t, \pi}\left[\int_{t}^{T} \exp \left(\left(\mu \bar{m}+\frac{\mu \bar{\sigma}^{2}}{2}\right)(s-t)\right)\right. \\
&\left.\quad \times \exp \left(\left(\mu \bar{m}+\frac{\mu \bar{\sigma}^{2}}{2}\right)(T-s)\right) n\left(\theta_{s}\right) \exp \left(-\int_{t}^{s} n\left(\theta_{u}\right) d u\right) d s\right] \\
&=\exp \left(\left(\mu \bar{m}+\frac{\mu(\bar{\sigma})^{2}}{2}\right)(T-t)\right) E^{t, \pi}\left[1_{\left\{\tau_{1} \leq T\right\}}\right]
\end{aligned}
$$

by using Jensen's inequality. On the other hand, we obtain

$$
\begin{aligned}
I_{1} \leq & E^{t, \pi}\left[\int_{t}^{T} \int_{\mathbb{R}^{m}}\left(h^{0} \exp \left(r_{0}(s-t)\right)+\sum_{i=1}^{m} h^{i} \exp \left(z^{i}\right)\right)^{\mu} \rho_{t, s}^{\theta}(z) d z\right. \\
& \left.\times \exp \left(\left(\mu \underline{m}+\frac{(\mu \bar{\sigma})^{2}}{2}\right)(T-s)\right) n\left(\theta_{s}\right) \exp \left(-\int_{t}^{s} n\left(\theta_{u}\right) d u\right) d s\right] \\
\leq & E^{t, \pi}\left[\int_{t}^{T}\left(h^{0} \exp \left(\mu r_{0}(s-t)\right)+\sum_{i=1}^{m} h^{i} \int_{\mathbb{R}^{m}} \exp \left(\mu z^{i}\right) \rho_{t, s}^{\theta}(z) d z\right)\right. \\
& \left.\times \exp \left(\left(\mu \underline{m}+\frac{(\mu \bar{\sigma})^{2}}{2}\right)(T-s)\right) n\left(\theta_{s}\right) \exp \left(-\int_{t}^{s} n\left(\theta_{u}\right) d u\right) d s\right] \\
\leq & E^{t, \pi}\left[\int_{t}^{T} \exp \left(\left(\mu \underline{m}+\frac{(\mu \bar{\sigma})^{2}}{2}\right)(s-t)\right)\right. \\
& \left.\times \exp \left(\left(\mu \underline{m}+\frac{(\mu \bar{\sigma})^{2}}{2}\right)(T-s)\right) n\left(\theta_{s}\right) \exp \left(-\int_{t}^{s} n\left(\theta_{u}\right) d u\right) d s\right] \\
= & \exp \left(\left(\mu \underline{m}+\frac{(\mu \bar{\sigma})^{2}}{2}\right)(T-t)\right) E^{t, \pi}\left[1_{\left\{\tau_{1} \leq T\right\}}\right],
\end{aligned}
$$

again by using Jensen's inequality and (5.2). 
(ii) (estimate (3.19)). By using Jensen's inequality, we have

$$
\begin{aligned}
I_{2}= & E^{t, \pi}\left[\int _ { \mathbb { R } _ { m } } \left(h^{0} \exp \left(r_{0}(T-t)\right)\right.\right. \\
& \left.\left.\quad+\sum_{i=1}^{m} h^{i} \exp \left(z^{i}\right)\right)^{\mu} \rho_{t, T}^{\theta}(z) d z \int_{T}^{\infty} n\left(\theta_{s}\right) \exp \left(-\int_{t}^{s} n\left(\theta_{u}\right) d u\right) d s\right] \\
\geq & E^{t, \pi}\left[\left(h^{0} \exp \left(r_{0}(T-t)\right)\right.\right. \\
& \left.\left.\quad+\sum_{i=1}^{m} h^{i} \int_{\mathbb{R}^{m}} \exp \left(z^{i}\right) \rho_{t, T}^{\theta}(z) d z\right)^{\mu} \int_{T}^{\infty} n\left(\theta_{s}\right) \exp \left(-\int_{t}^{s} n\left(\theta_{u}\right) d u\right) d s\right] \\
\geq & E^{t, \pi}\left[\left\{\sum_{i=0}^{m} h^{i} \exp \left(\left(\bar{m}+\frac{(\bar{\sigma})^{2}}{2}\right)(T-t)\right\}^{\mu} \int_{T}^{\infty} n\left(\theta_{s}\right) \exp \left(-\int_{t}^{s} n\left(\theta_{u}\right) d u\right) d s\right]\right. \\
\geq & E^{t, \pi}\left[\exp \left(\left(\bar{m}+\frac{(\bar{\sigma})^{2}}{2}\right)(T-t)\right)^{\mu} \int_{T}^{\infty} n\left(\theta_{s}\right) \exp \left(-\int_{t}^{s} n\left(\theta_{u}\right) d u\right) d s\right] \\
= & \exp \left(\left(\mu \bar{m}+\frac{\mu(\bar{\sigma})^{2}}{2}\right)(T-t)\right) E^{t, \pi}\left[1_{\left\{\tau_{1}>T\right\}}\right],
\end{aligned}
$$

from (5.2), since the function $x^{\mu}$ is decreasing. On the other hand, by using Jensen's inequality, we have

$$
\begin{aligned}
I_{2}= & E^{t, \pi}\left[\int _ { \mathbb { R } ^ { m } } \left(h^{0} \exp \left(r_{0}(T-t)\right)\right.\right. \\
& \left.\left.\quad+\sum_{i=1}^{m} h^{i} \exp \left(z^{i}\right)\right)^{\mu} \rho_{t, T}^{\theta}(z) d z \int_{T}^{\infty} n\left(\theta_{s}\right) \exp \left(-\int_{t}^{s} n\left(\theta_{u}\right) d u\right) d s\right] \\
\leq \quad & E^{t, \pi}\left[h^{0} \exp \left(\mu r_{0}(T-t)\right)\right. \\
& \left.\quad+\sum_{i=1}^{m} h^{i} \int_{\mathbb{R}^{m}} \exp \left(\mu z^{i}\right) \rho_{t, T}^{\theta}(z) d z \int_{T}^{\infty} n\left(\theta_{s}\right) \exp \left(-\int_{t}^{s} n\left(\theta_{u}\right) d u\right) d s\right] \\
= & E^{t, \pi}\left[\sum_{i=0}^{m} h^{i} \exp \left(\mu \int_{t}^{T} m_{i}\left(\theta_{s}\right) d s+\frac{\mu^{2}}{2} \int_{t}^{T} \sigma_{i}^{2}\left(\theta_{s}\right) d s\right)\right. \\
& \left.\quad \times \int_{T}^{\infty} n\left(\theta_{s}\right) \exp \left(-\int_{t}^{s} n\left(\theta_{u}\right) d u\right) d s\right] \\
\leq \quad & E^{t, \pi}\left[\exp \left(\left(\mu \underline{m}+\frac{(\mu \bar{\sigma})^{2}}{2}\right)(T-t)\right) \int_{T}^{\infty} n\left(\theta_{s}\right) \exp \left(-\int_{t}^{s} n\left(\theta_{u}\right) d u\right) d s\right] \\
= & \exp \left(\left(\mu \underline{m}+\frac{(\mu \bar{\sigma})^{2}}{2}\right)(T-t)\right) E^{t, \pi}\left[1_{\left\{\tau_{1}>T\right\}}\right],
\end{aligned}
$$

because of (5.2).

(iii) (estimate (3.20)). Since

$$
\hat{\xi}(t, \pi, h ; g)=\frac{1}{\mu} \log \left(I_{1}+I_{2}\right) .
$$

The estimate (3.20) follows from $(i)$ and $(i i)$.

Lemma 5.4. For all $g \in \mathcal{G}$, the function $\exp (\mu \hat{\xi}(t, \pi, h ; g))$ is continuous with respect to $h$. Furthermore, for each $g \in \mathcal{G}_{1}$ the relation (3.21) holds and for each $g \in \mathcal{G}_{2}$ the relation (3.22) holds. 
Proof. Let us first prove the continuity of $\exp (\mu \hat{\xi}(t, \pi, h ; g))$. From (5.13), we have for $m \geq 1$

$$
\exp \left(\mu D\left(h, X_{T \wedge \tau_{1}}-X_{t}\right)+\mu g\left(\tau_{1}, \pi_{1}\right)\right) \leq \sum_{i=0}^{m} \exp \left(\mu\left(X_{T \wedge \tau_{1}}^{i}-X_{t}^{i}\right)+\mu g\left(\tau_{1}, \pi_{1}\right)\right) .
$$

Similarly to (5.15), we have for each $i$

$$
E^{t, \pi}\left[\exp \left(\mu\left(X_{T \wedge \tau_{1}}^{i}-X_{t}^{i}\right)+\mu g\left(\tau_{1}, \pi_{1}\right)\right)\right]<\infty .
$$

Applying the dominated convergence theorem, for $h_{n} \subset \bar{H}_{m}$, s.t. $\lim _{n \rightarrow \infty} h_{n}=h \in \bar{H}_{m}$

$$
\begin{aligned}
& \lim _{n \rightarrow \infty} \exp \left(\mu \hat{\xi}\left(t, \pi, h_{n} ; g\right)\right) \\
= & E^{t, \pi}\left[\lim _{n \rightarrow \infty} \exp \left(\mu D\left(h_{n}, X_{T \wedge \tau_{1}}-X_{t}\right)+\mu g\left(\tau_{1}, \pi_{1}\right) 1_{\left\{\tau_{1} \leq T\right\}}\right)\right] \\
= & \exp (\mu \hat{\xi}(t, \pi, h ; g)) .
\end{aligned}
$$

We next prove that, for $g \in \mathcal{G}_{1}$, the relation (3.21) holds. For this purpose, recalling Corollary 2.2 , we rewrite

$$
\begin{aligned}
& \exp (\mu \hat{\xi}(t, \pi, h ; g)) \\
& =E^{t, \pi}\left[\exp \left(\mu D\left(h, X_{T \wedge \tau_{1}}-X_{t}\right)+\mu g\left(\tau_{1}, M\left(\tau_{1}-t, X_{\tau_{1}}-X_{t}, \pi\right)\right) 1_{\left\{\tau_{1} \leq T\right\}}\right)\right] \\
& =\sum_{j} E^{t, e_{j}}\left[\operatorname { e x p } \left(\mu D\left(h, X_{T \wedge \tau_{1}}-X_{t}\right)\right.\right. \\
& \left.\left.+\mu g\left(\tau_{1}, M\left(\tau_{1}-t, X_{\tau_{1}}-X_{t}, \pi\right)\right) 1_{\left\{\tau_{1} \leq T\right\}}\right)\right] \pi^{j}
\end{aligned}
$$

Then, recalling the Definition 3.3 of $\hat{\xi}(\cdot)$, from (3.20) in Proposition 3.2 and (2.4) it follows that

$$
\begin{aligned}
& |\exp (\mu \hat{\xi}(t, \pi, h ; g))-\exp (\mu \hat{\xi}(t, \bar{\pi}, h ; g))| \\
= & \mid \sum_{j} E^{t, e_{j}}\left[\operatorname { e x p } \left(\mu D\left(h, X_{T \wedge \tau_{1}}-X_{t}\right)\right.\right. \\
& \left.\left.+\mu g\left(\tau_{1}, M\left(\tau_{1}-t, X_{\tau_{1}}-X_{t}, \pi\right)\right) 1_{\left\{\tau_{1} \leq T\right\}}\right)\right]\left(\pi^{j}-\bar{\pi}^{j}\right) \\
& +\sum_{j} E^{t, e_{j}}\left[\operatorname { e x p } ( \mu D ( h , X _ { \tau _ { 1 } } - X _ { t } ) ) \left\{\exp \left(\mu g\left(\tau_{1}, M\left(\tau_{1}-t, X_{\tau_{1}}-X_{t}, \pi\right)\right)\right)\right.\right. \\
& \left.\left.\left.-\exp \left(\mu g\left(\tau_{1}, M\left(\tau_{1}-t, X_{\tau_{1}}-X_{t}, \bar{\pi}\right)\right)\right)\right\} 1_{\left\{\tau_{1} \leq T\right\}}\right)\right] \bar{\pi}^{j} \mid \\
\leq & \exp \left(\left(\mu \underline{m}+\frac{(\mu \bar{\sigma})^{2}}{2}\right)(T-t)\right) \frac{2}{\log 3} d_{H}(\pi, \bar{\pi}) \\
& +E^{t, \bar{\pi}}\left[\exp \left(\mu D\left(h, X_{1}-X_{t}\right)\right) \mid \exp \left(\mu g\left(\tau_{1}, M\left(\tau_{1}-t, X_{\tau_{1}}-X_{t}, \pi\right)\right)\right)\right. \\
& \left.\left.-\exp \left(\mu g\left(\tau_{1}, M\left(\tau_{1}-t, X_{\tau_{1}}-X_{t}, \bar{\pi}\right)\right)\right) \mid 1_{\left\{\tau_{1} \leq T\right\}}\right)\right] .
\end{aligned}
$$


Furthermore, by the definition the definition of $\mathcal{G}_{1}$ (see (3.15) in Definition 3.2), using also (3.10)

$$
\begin{aligned}
& \mid \exp \left(\mu g\left(\tau_{1}, M\left(\tau_{1}-t, X_{\tau_{1}}-X_{t}, \pi\right)\right)\right) \\
& -\exp \left(\mu g\left(\tau_{1}, M\left(\tau_{1}-t, X_{\tau_{1}}-X_{t}, \bar{\pi}\right)\right)\right) \mid \\
& \leq \exp \left(\left(\mu \underline{m}+\frac{(\mu \bar{\sigma})^{2}}{2}\right)\left(T-\tau_{1}\right)\right) \\
& \times \frac{2}{\log 3} d_{H}\left(M\left(\tau_{1}-t, X_{\tau_{1}}-X_{t}, \pi\right), M\left(\tau_{1}-t, X_{\tau_{1}}-X_{t}, \bar{\pi}\right)\right) \\
& \leq \frac{2}{\log 3} d_{H}(\pi, \bar{\pi}) \exp \left(\left(\mu \underline{m}+\frac{(\mu \bar{\sigma})^{2}}{2}\right)\left(T-\tau_{1}\right)\right) .
\end{aligned}
$$

Therefore, we obtain

$$
\begin{aligned}
& |\exp (\mu \hat{\xi}(t, \pi, h ; g))-\exp (\mu \hat{\xi}(t, \bar{\pi}, h ; g))| \\
\leq & \frac{2}{\log 3} d_{H}(\pi, \bar{\pi}) \exp \left(\left(\mu \underline{m}+\frac{(\mu \bar{\sigma})^{2}}{2}\right)(T-t)\right)(1+c) .
\end{aligned}
$$

Finally, to prove that for $g \in \mathcal{G}_{2}$ the relation (3.22) holds, we rewrite, using the time homogeneity of $\left(X_{t}, \theta_{t}\right)$,

$$
\begin{aligned}
& \exp (\mu \hat{\xi}(t, \pi, h ; g)) \\
= & E^{t, \pi}\left[\exp \left(\mu D\left(h, X_{T}-X_{t}\right)\right) 1_{\left\{\tau_{1}>T\right\}}+\exp \left(\mu D\left(h, X_{\tau_{1}}-X_{t}\right)+\mu g\left(\tau_{1}, \pi_{1}\right)\right) 1_{\left\{\tau_{1} \leq T\right\}}\right] \\
= & E^{t, \pi}\left[\exp \left(\mu D\left(h, X_{T-t}-X_{0}\right)\right) 1_{\left\{\tau_{1}>T-t\right\}}\right. \\
& \left.+\exp \left(\mu D\left(h, X_{\tau_{1}}-X_{0}\right)+\mu g\left(\tau_{1}+t, \pi_{1}\right)\right) 1_{\left\{\tau_{1} \leq T-t\right\}}\right] .
\end{aligned}
$$

Therefore, recalling that $\bar{t}<t$,

$$
\begin{aligned}
& |\exp (\mu \hat{\xi}(t, \pi, h ; g))-\exp (\mu \hat{\xi}(\bar{t}, \pi, h ; g))| \\
\leq & \left|E^{0, \pi}\left[\exp \left(\mu D\left(h, X_{T-t}-X_{0}\right)\right)\left\{1_{\left\{\tau_{1}>T-t\right\}}-1_{\left\{\tau_{1}>T-\bar{t}\right\}}\right\}\right]\right| \\
& +\left|E^{0, \pi}\left[\exp \left(\mu D\left(h, X_{\tau_{1}}-X_{0}\right)+\mu g\left(\tau_{1}+t, \pi_{1}\right)\right)\left\{1_{\left\{\tau_{1} \leq T-t\right\}}-1_{\left\{\tau_{1} \leq T-\bar{t}\right\}}\right\}\right]\right| \\
& +\mid E^{0, \pi}\left[\left\{\exp \left(\mu D\left(h, X_{T-t}-X_{0}\right)-\exp \left(\mu D\left(h, X_{T-\bar{t}}-X_{0}\right)\right)\right\} 1_{\left\{\tau_{1}>T-\bar{t}\right\}}\right] \mid\right. \\
& +\mid E^{0, \pi}\left[\exp \left(\mu D\left(h, X_{\tau_{1}}-X_{0}\right)\right)\left\{\exp \left(\mu g\left(\tau_{1}+t, \pi_{1}\right)\right)-\exp \left(\mu g\left(\tau_{1}+\bar{t}, \pi_{1}\right)\right\} 1_{\left\{\tau_{1} \leq T-\bar{t}\right\}}\right] \mid\right. \\
\equiv & J_{1}+J_{2}+J_{3}+J_{4} .
\end{aligned}
$$

Now we have

$$
\begin{aligned}
& J_{1} \leq \exp \left(\left(\mu \underline{m}+\frac{(\mu \bar{\sigma})^{2}}{2}\right)(T-t)\right) P^{0, \pi}\left(T-t<\tau_{1}<T-\bar{t}\right) \\
= & \exp \left(\left(\mu \underline{m}+\frac{(\mu \bar{\sigma})^{2}}{2}\right)(T-t)\right) E^{t, \pi}\left[\int_{T-t}^{T-\bar{t}} n\left(\theta_{s}\right) \exp \left(\int_{0}^{s}-n\left(\theta_{u}\right) d u\right) d s\right] \\
\leq & \exp \left(\left(\mu \underline{m}+\frac{(\mu \bar{\sigma})^{2}}{2}\right)(T-t)\right) \int_{T-t}^{T-\bar{t}} \bar{n} \exp (-\underline{n} s) d s \\
\leq & \exp \left(\left(\mu \underline{m}+\frac{(\mu \bar{\sigma})^{2}}{2}\right)(T-t)\right)\left(\frac{\bar{n}}{\underline{n}}\right)\left(e^{\underline{n}(t-\bar{t})}-1\right) .
\end{aligned}
$$

We also have, using (3.20),

$$
J_{2} \leq \exp \left(\left(\mu \underline{m}+\frac{(\mu \bar{\sigma})^{2}}{2}\right)(T-t)\right)\left(\frac{\bar{n}}{\underline{n}}\right)\left(e^{\underline{n}(t-\bar{t})}-1\right) .
$$


Further, since $|D(h, x)-D(h, y)| \leq|x-y|$ holds from (5.10), we obtain

$$
\begin{aligned}
J_{3} \leq & E^{0, \pi}\left[\left|\exp \left(\mu D\left(h, X_{T-t}-X_{0}\right)\right)-\exp \left(\mu D\left(h, X_{T-\bar{t}}-X_{0}\right)\right)\right|\right] \\
= & E^{0, \pi}\left[\exp \left(\mu D\left(h, X_{T-t}-X_{0}\right)\right)\right. \\
& \left.\times\left|1-\exp \left(\mu\left(D\left(h, X_{T-\bar{t}}-X_{0}\right)-D\left(h, X_{T-t}-X_{0}\right)\right)\right)\right|\right] \mid \\
\leq & E^{0, \pi}\left[\exp \left(\mu D\left(h, X_{T-t}-X_{0}\right)\right)\left|1-\exp \left(\mu\left|X_{T-\bar{t}}-X_{T-t}\right|\right)\right|\right] \\
= & E^{0, \pi}\left[\exp \left(\mu D\left(h, X_{T-t}-X_{0}\right)\right) E\left[\left|1-\exp \left(\mu\left|X_{T-\bar{t}}-X_{T-t}\right|\right)\right| \mid X_{T-t}\right]\right] .
\end{aligned}
$$

Since $\left(X_{t}, \theta_{t}\right)$ is a time homogeneous process, we have

$$
\begin{aligned}
& E\left[\mid 1-\exp \left(\mu\left|X_{T-\bar{t}}-X_{T-t}\right|\right) \| X_{T-t}\right] \\
= & E\left[\mid 1-\exp \left(\mu \mid \int_{T-t}^{T-\bar{t}} r\left(\theta_{s}\right)-d\left(\sigma \sigma^{*}\left(\theta_{s}\right)\right) d s+\int_{T-t}^{T-\bar{t}} \sigma\left(\theta_{s}\right) d B_{s}\right) \| X_{T-t}\right] \\
= & E_{X_{T-t}}\left[\left|1-\exp \left(\mu\left|\int_{T-t}^{T-\bar{t}} r\left(\theta_{s}\right)-d\left(\sigma \sigma^{*}\left(\theta_{s}\right)\right) d s+\int_{T-t}^{T-\bar{t}} \sigma\left(\theta_{s}\right) d B_{s}\right|\right)\right|\right] \\
= & E_{X_{T-t}}\left[\left|1-\exp \left(\mu\left|\int_{0}^{t-\bar{t}} r\left(\theta_{s}\right)-d\left(\sigma \sigma^{*}\left(\theta_{s}\right)\right) d s+\int_{0}^{t-\bar{t}} \sigma\left(\theta_{s}\right) d B_{s}\right|\right)\right|\right] \\
= & E_{X_{T-t}}\left[\mid 1-\exp \left(\mu\left|X_{t-\bar{t}}-X_{0}\right|\right)\right] \\
= & l(t-\bar{t}),
\end{aligned}
$$

where $l$ is the function defined in (3.1). Hence, we obtain

$$
\begin{aligned}
J_{3} & \leq E^{0, \pi}\left[\exp \left(\mu D\left(h, X_{T-t}-X_{0}\right)\right)\right] l(t-\bar{t}) \\
& \leq \exp \left(\left(\mu \underline{m}+\frac{(\mu \bar{\sigma})^{2}}{2}\right)(T-t)\right) l(t-\bar{t}) .
\end{aligned}
$$

Since $g \in \mathcal{G}_{2}$, we have

$$
\begin{aligned}
J_{4} \leq & E^{0, \pi}\left[\exp \left(\mu D\left(h, X_{\tau_{1}}-X_{0}\right)\right)\right. \\
& \left.\left.\exp \left(\left(\mu \underline{m}+\frac{(\mu \bar{\sigma})^{2}}{2}\right)\left(T-t-\tau_{1}\right)\right) k(t-\bar{t})\right) 1_{\left\{\tau_{1} \leq T-\bar{t}\right\}}\right] \\
\leq & c \exp \left(\left(\mu \underline{m}+\frac{(\mu \bar{\sigma})^{2}}{2}\right)(T-t)\right) k(t-\bar{t}),
\end{aligned}
$$

by using (3.18) in Proposition 3.2.

Putting all the estimates together, we finally obtain

$$
\begin{aligned}
& |\exp (\mu \hat{\xi}(t, \pi, h ; g))-\exp (\mu \hat{\xi}(\bar{t}, \pi, h ; g))| \\
\leq & \exp \left(\left(\mu \underline{m}+\frac{(\mu \bar{\sigma})^{2}}{2}\right)(T-t)\right)\left(2\left(\frac{\bar{n}}{\underline{n}}\right)\left(e^{n(t-\bar{t})}-1\right)+l(t-\bar{t})+c k(t-\bar{t})\right) .
\end{aligned}
$$

Proof of Proposition 3.5. The equality (3.46) is shown in Lemma 5.5 below. This lemma is followed by Lemma 5.6 that is preliminary to Lemma 5.7, from which then (3.47) follows.

Lemma 5.5. For each $n \geq 0$, the equality (3.46) holds. 
Proof. By definition we have

$$
\bar{W}^{0}(t, \pi)=W^{0}(t, \pi) .
$$

Moreover, $\bar{W}^{0}(t, \pi) \in \mathcal{G}_{1} \cap \mathcal{G}_{2}$ because of Proposition 3.3. Therefore, in Corollary 3.1, we set $g(t, \pi)=\bar{W}^{0}(t, \pi)$ and obtain a Borel function $\hat{h}^{(n)}(t, \pi)$ satisfying (3.31) for $n \geq 0$. Then,

$$
\bar{W}^{n}(t, \pi)=J_{\mu}^{n} \bar{W}^{0}(t, \pi)=\sup _{h \in H_{m}} \hat{\xi}\left(t, \pi, h ; J_{\mu}^{n-1} \bar{W}^{0}\right)=\hat{\xi}\left(t, \pi, \hat{h}^{(n-1)}(t, \pi) ; J_{\mu}^{n-1} \bar{W}^{0}\right) .
$$

We also have a Borel function $\bar{h}(t, \pi)$ such that

$$
\bar{W}^{0}(t, \pi)=\sup _{h} \bar{W}^{0}(t, \pi, h)=\bar{W}^{0}(t, \pi, \bar{h}(t, \pi)) .
$$

We define a strategy $\bar{h}^{(n)} \in \mathcal{A}^{n}$ as follows.

$$
\begin{aligned}
& \bar{h}_{k}^{(n)}=\hat{h}^{(n-1-k)}\left(\tau_{k}, \pi_{k}\right), k=0, \ldots, n-1, \\
& \bar{h}_{n}^{(n)}=\bar{h}\left(\tau_{n}, \pi_{n}\right), \\
& \bar{h}_{k}^{(n)}=\gamma\left(\tilde{X}_{\tau_{k}}-\tilde{X}_{\tau_{n}}, \bar{h}_{n}^{(n)}\right), k \geq n+1 .
\end{aligned}
$$

First, to show that $\bar{W}_{n}(t, \pi) \leq W_{n}(t, \pi)$, we rewrite $\bar{W}^{n}$ as follows,

$$
\begin{aligned}
& \bar{W}^{n}(t, \pi) \\
= & \sup _{h \in \bar{H}_{m}} \hat{\xi}\left(t, \pi, h ; J_{\mu}^{n-1} \bar{W}^{0}\right) \\
= & \hat{\xi}\left(t, \pi, \hat{h}^{(n-1)}(t, \pi) ; J_{\mu}^{n-1} \bar{W}^{0}\right) \\
= & \frac{1}{\mu} \log E^{t, \pi}\left[\exp \left(\mu D\left(\hat{h}^{(n-1)}(t, \pi), X_{T \wedge \tau_{1}}-X_{t}\right)+\mu \bar{W}^{n-1}\left(\tau_{1}, \pi_{1}\right) 1_{\left\{\tau_{1} \leq T\right\}}\right)\right] \\
= & \frac{1}{\mu} \log E^{t, \pi}\left[\exp \left(\mu D\left(\hat{h}^{(n-1)}(t, \pi), X_{T}-X_{t}\right)\right) 1_{\left\{\tau_{1}>T\right\}}\right. \\
& \left.\quad+\exp \left(\mu D\left(\hat{h}^{(n-1)}(t, \pi), X_{\tau_{1}}-X_{t}\right)+\mu \bar{W}^{n-1}\left(\tau_{1}, \pi_{1}\right)\right) 1_{\left\{\tau_{1} \leq T\right\}}\right] \\
= & \frac{1}{\mu} \log E^{t, \pi}\left[e^{\mu D\left(\hat{h}^{(n-1)}(t, \pi), X_{T}-X_{t}\right)} 1_{\left\{\tau_{1}>T\right\}}+e^{\mu D\left(\hat{h}^{(n-1)}(t, \pi), X_{\tau_{1}}-X_{t}\right)}\right. \\
& \quad \cdot E^{\tau_{1}, \pi_{1}}\left[e^{\left.\left.\mu D\left(\hat{h}^{(n-2)}\left(\tau_{1}, \pi_{1}\right), X_{T \wedge \tau_{2}}-X_{\tau_{1}}\right)+\mu \bar{W}^{n-2}\left(\tau_{2}, \pi_{2}\right) 1_{\left\{\tau_{2} \leq T\right\}}\right] 1_{\left\{\tau_{1} \leq T\right\}}\right]}\right.
\end{aligned}
$$

Noting that

$$
\begin{gathered}
E^{t, \pi}\left[e^{\mu D\left(\hat{h}^{(n-1)}(t, \pi), X_{\tau_{1}}-X_{t}\right)}\right. \\
\cdot E^{\tau_{1}, \pi_{1}}\left[e^{\left.\left.\mu D\left(\hat{h}^{(n-2)}\left(\tau_{1}, \pi_{1}\right), X_{T \wedge \tau_{2}}-X_{\tau_{1}}\right)+\mu \bar{W}^{n-2}\left(\tau_{2}, \pi_{2}\right) 1_{\left\{\tau_{2} \leq T\right\}}\right] 1_{\left\{\tau_{1} \leq T\right\}}\right]}\right. \\
=E^{t, \pi}\left[e^{\mu D\left(\hat{h}^{(n-1)}(t, \pi), X_{\tau_{1}}-X_{t}\right)} E^{\tau_{1}, \pi_{1}}\left[e^{\mu D\left(\hat{h}^{(n-2)}\left(\tau_{1}, \pi_{1}\right), X_{T}-X_{\tau_{1}}\right)} 1_{\left\{\tau_{2}>T\right\}}\right] 1_{\left\{\tau_{1} \leq T\right\}}\right] \\
+E^{t, \pi}\left[e^{\mu D\left(\hat{h}^{(n-1)}(t, \pi), X_{\tau_{1}}-X_{t}\right)}\right. \\
\left.\cdot E^{\tau_{1}, \pi_{1}}\left[e^{\mu D\left(\hat{h}^{(n-2)}\left(\tau_{1}, \pi_{1}\right), X_{\tau_{2}}-X_{\tau_{1}}\right)+\mu \bar{W}^{n-2}\left(\tau_{2}, \pi_{2}\right)} 1_{\left\{\tau_{2} \leq T\right\}}\right] 1_{\left\{\tau_{1} \leq T\right\}}\right]
\end{gathered}
$$


we have

$$
\begin{aligned}
& \bar{W}^{n}(t, \pi) \\
& =\frac{1}{\mu} \log E^{t, \pi}\left[e^{\left.\mu D\left(\hat{h}^{(n-1)}(t, \pi), X_{T \wedge \tau_{1}}-X_{t}\right)+\mu D\left(\hat{h}^{(n-2)}\left(\tau_{1}, \pi_{1}\right), X_{T \wedge \tau_{2}}-X_{\tau_{1}}\right) 1_{\left\{\tau_{1} \leq T\right\}}+\mu \bar{W}^{n-2}\left(\tau_{2}, \pi_{2}\right) 1_{\left\{\tau_{2} \leq T\right\}}\right]}\right. \\
& =\frac{1}{\mu} \log E^{t, \pi}\left[\operatorname { e x p } \left(\mu \sum_{k=1}^{n} D\left(\bar{h}^{(n)}\left(\tau_{k-1}, \pi_{k-1}\right), X_{T \wedge \tau_{k}}-X_{\tau_{k-1}}\right) 1_{\left\{\tau_{k-1} \leq T\right\}}\right.\right. \\
& \left.\left.\quad+\mu \bar{W}^{0}\left(\tau_{n}, \pi_{n}, \bar{h}^{(n)}\left(\tau_{n}, \pi_{n}\right)\right) 1_{\left\{\tau_{n}<T\right\}}\right)\right],
\end{aligned}
$$

inductively. By Corollary 3.3 we then have

$$
\begin{aligned}
& \bar{W}^{n}(t, \pi) \\
= & \frac{1}{\mu} \log E^{t, \pi}\left[\operatorname { e x p } \left(\mu \sum_{k=1}^{n} D\left(\bar{h}_{n}\left(\tau_{k-1}, \pi_{k-1}\right), X_{T \wedge \tau_{k}}-X_{\tau_{k-1}}\right) 1_{\left\{\tau_{k-1}<T\right\}}\right.\right. \\
& \left.\left.\quad+\mu \bar{W}^{0}\left(\tau_{n}, \pi_{n}, \bar{h}_{n}\left(\tau_{n}, \pi_{n}\right)\right) 1_{\left\{\tau_{n}<T\right\}}\right)\right] \\
\leq & \sup _{h \in \mathcal{A}^{n}} \frac{1}{\mu} \log E^{t, \pi}\left[\operatorname { e x p } \left(\mu \sum_{k=1}^{n} D\left(h_{k-1}, X_{T \wedge \tau_{k}}-X_{\tau_{k-1}}\right) 1_{\left\{\tau_{k-1}<T\right\}}\right.\right. \\
\quad & \left.\left.\quad+\mu \bar{W}^{0}\left(\tau_{n}, \pi_{n}, h_{n}\right) 1_{\left\{\tau_{n} \leq T\right\}}\right)\right] \\
= & W^{n}(t, \pi) .
\end{aligned}
$$

Next, we shall prove the converse inequality. By applying Lemma 3.4, we have for $h \in \mathcal{A}^{n}$

$$
\begin{aligned}
& W(t, \pi, h .) \\
& =\frac{1}{\mu} \log E^{t, \pi}\left[\exp \left(\mu \sum_{k=1}^{n} D\left(h_{k-1}, X_{T \wedge \tau_{k}}-X_{\tau_{k-1}}\right) 1_{\left\{\tau_{k-1}<T\right\}}+\mu \bar{W}^{0}\left(\tau_{n}, \pi_{n}, h_{n}\right) 1_{\left\{\tau_{n} \leq T\right\}}\right)\right] \\
& \leq \frac{1}{\mu} \log E^{t, \pi}\left[\exp \left(\mu \sum_{k=1}^{n} D\left(h_{k-1}, X_{T \wedge \tau_{k}}-X_{\tau_{k-1}}\right) 1_{\left\{\tau_{k-1}<T\right\}}+\mu \bar{W}^{0}\left(\tau_{n}, \pi_{n}\right) 1_{\left\{\tau_{n} \leq T\right\}}\right)\right] \\
& =\frac{1}{\mu} \log E^{t, \pi}\left[\exp \left(\mu \sum_{k=1}^{n-1} D\left(h_{k-1}, X_{\tau_{k}}-X_{\tau_{k-1}}\right) 1_{\left\{\tau_{k-1}<T\right\}}\right)\right. \\
& \times \exp \left(\mu D\left(h_{n-1}, X_{T \wedge \tau_{n}}-X_{\tau_{n-1}}\right)+\mu \bar{W}^{0}\left(\tau_{n}, \pi_{n}\right) 1_{\left\{\tau_{n} \leq T\right\}}\right) 1_{\left\{\tau_{n-1} \leq T\right\}} \\
& \left.+\exp \left(\mu \sum_{k=1}^{n-1} D\left(h_{k-1}, X_{T \wedge \tau_{k}}-X_{\tau_{k-1}}\right) 1_{\left\{\tau_{k-1}<T\right\}}\right) 1_{\left\{\tau_{n-1}>T\right\}}\right] \\
& =\frac{1}{\mu} \log E^{t, \pi}\left[\exp \left(\mu \sum_{k=1}^{n-1} D\left(h_{k-1}, X_{\tau_{k}}-X_{\tau_{k-1}}\right) 1_{\left\{\tau_{k-1}<T\right\}}\right)\right. \\
& \times E^{\tau_{n-1}, \pi_{n-1}}\left[\exp \left(\mu D\left(h_{n-1}, X_{T \wedge \tau_{n}}-X_{\tau_{n-1}}\right)+\mu \bar{W}^{0}\left(\tau_{n}, \pi_{n}\right) 1_{\left\{\tau_{n} \leq T\right\}}\right)\right] 1_{\left\{\tau_{n-1} \leq T\right\}} \\
& \left.+\exp \left(\mu \sum_{k=1}^{n-1} D\left(h_{k-1}, X_{T \wedge \tau_{k}}-X_{\tau_{k-1}}\right) 1_{\left\{\tau_{k-1}<T\right\}}\right) 1_{\left\{\tau_{n-1}>T\right\}}\right] .
\end{aligned}
$$


By the definition of $\hat{\xi}$ and $\bar{W}^{1}$ we have

$$
\begin{aligned}
& E^{\tau_{n-1}, \pi_{n-1}}\left[\exp \left(\mu D\left(h_{n-1}, X_{T \wedge \tau_{n}}-X_{\tau_{n-1}}\right)+\mu \bar{W}^{0}\left(\tau_{n}, \pi_{n}\right) 1_{\left\{\tau_{n} \leq T\right\}}\right)\right] \\
= & \exp \left(\mu \hat{\xi}\left(\tau_{n-1}, \pi_{n-1}, h_{n-1} ; \bar{W}^{0}\right)\right) \\
\geq & \exp \left(\mu \sup _{h \in \bar{H}_{m}} \hat{\xi}\left(\tau_{n-1}, \pi_{n-1}, h ; \bar{W}^{0}\right)\right)=\exp \left(\mu \bar{W}^{1}\left(\tau_{n-1}, \pi_{n-1}\right)\right) .
\end{aligned}
$$

Therefore, for $h \in \mathcal{A}^{n}$, we have inductively

$$
\begin{aligned}
& W(t, \pi, h .) \\
\leq & \frac{1}{\mu} \log E^{t, \pi}\left[\exp \left(\mu \sum_{k=1}^{n-1} D\left(h_{k-1}, X_{T \wedge \tau_{k}}-X_{\tau_{k-1}}\right) 1_{\left\{\tau_{k-1}<T\right\}}+\mu \bar{W}^{1}\left(\tau_{n}, \pi_{n}\right) 1_{\left\{\tau_{n} \leq T\right\}}\right)\right] \\
\leq & \frac{1}{\mu} \log E^{t, \pi}\left[\exp \left(\mu D\left(h, X_{T \wedge \tau_{1}}-X_{0}\right)+\mu \bar{W}^{n-1}\left(\tau_{1}, \pi_{1}\right) 1_{\left\{\tau_{1} \leq T\right\}}\right)\right] \\
\leq & \bar{W}^{n}(t, \pi) .
\end{aligned}
$$

Lemma 5.6. For all $h \in \mathcal{A}$, we have

$$
\begin{array}{ll}
\lim _{n \rightarrow \infty} \frac{1}{\mu} \log E^{t, \pi}[ & \exp \left(\mu \sum_{k=1}^{n} D\left(h_{k-1}, X_{T \wedge \tau_{k}}-X_{\tau_{k-1}}\right) 1_{\left\{\tau_{k-1}<T\right\}}\right. \\
& \left.\left.+\mu W^{0}\left(\tau_{n}, \pi_{n}, h_{n}\right) 1_{\left\{\tau_{n} \leq T\right\}}\right)\right] \\
=W(t, \pi, h .) . &
\end{array}
$$

Proof.

$$
\begin{aligned}
& E^{t, \pi}\left[\operatorname { e x p } \left(\mu \sum_{k=1}^{n} D\left(h_{k-1}, X_{T \wedge \tau_{k}}-X_{\tau_{k-1}}\right) 1_{\left\{\tau_{k-1} \leq T\right\}}\right.\right. \\
& \left.\left.+\mu W^{0}\left(\tau_{n}, \pi_{n}, h_{n}\right) 1_{\left\{\tau_{n} \leq T\right\}}\right)\right] \\
= & E^{t, \pi}\left[\exp \left(\mu \sum_{k=1}^{n} D\left(h_{k-1}, X_{\tau_{k}}-X_{\tau_{k-1}}\right)+\mu W^{0}\left(\tau_{n}, \pi_{n}, h_{n}\right)\right) 1_{\left\{\tau_{n} \leq T\right\}}\right] \\
& +E^{t, \pi}\left[\exp \left(\mu \sum_{k=1}^{n} D\left(h_{k-1}, X_{T \wedge \tau_{k}}-X_{\tau_{k-1}}\right) 1_{\left\{\tau_{k-1} \leq T\right\}}\right) 1_{\left\{\tau_{n}>T\right\}}\right] \\
=: & I_{1}(n)+I_{2}(n) .
\end{aligned}
$$

We shall first give an estimate for $I_{1}(n)$. From (3.11) in Proposition 3.1 it follows that for $h \in \bar{H}_{m}$

$$
\exp \left(\mu W^{0}(t, \pi, h)\right) \leq \exp \left(\left(\mu \underline{m}+\frac{(\mu \bar{\sigma})^{2}}{2}\right)(T-t)\right)
$$


Therefore, we have

$$
\begin{aligned}
& I_{1}(n) \leq E^{t, \pi}\left[\exp \left(\mu \sum_{k=1}^{n} D\left(h_{k-1}, X_{\tau_{k}}-X_{\tau_{k-1}}\right)+\mu\left(\underline{m}+\frac{(\mu \bar{\sigma})^{2}}{2}\right)\left(T-\tau_{n}\right)\right) 1_{\left\{\tau_{n} \leq T\right\}}\right] \\
& \leq E^{t, \pi}\left[\operatorname { e x p } \left(\mu \sum _ { k = 1 } ^ { n - 1 } D ( h _ { k - 1 } , X _ { \tau _ { k } } - X _ { \tau _ { k - 1 } } ) E ^ { \tau _ { n - 1 } , \pi _ { n - 1 } } \left[\operatorname { e x p } \left(\mu D\left(h_{n-1}, X_{\tau_{n}}-X_{\tau_{n-1}}\right)\right.\right.\right.\right. \\
& \left.\left.\left.\quad+\mu\left(\underline{m}+\frac{(\mu \bar{\sigma})^{2}}{2}\right)\left(T-\tau_{n}\right)\right) 1_{\left\{\tau_{n}<T\right\}}\right] 1_{\left\{\tau_{n-1} \leq T\right\}}\right] \\
& \leq c E^{t, \pi}\left[\exp \left(\mu \sum_{k=1}^{n-1} D\left(h_{k-1}, X_{\tau_{k}}-X_{\tau_{k-1}}\right) \exp \left(\left(\mu \underline{m}+\frac{(\mu \bar{\sigma})^{2}}{2}\right)\left(T-\tau_{n-1}\right)\right) 1_{\left\{\tau_{n-1} \leq T\right\}}\right],\right.
\end{aligned}
$$

by using Proposition 3.2(i) because clearly $\exp \left(\left(\mu \underline{m}+\frac{(\mu \bar{\sigma})^{2}}{2}\right)(T-t)\right) \in \mathcal{G}$. Thus, we obtain inductively

$$
\begin{aligned}
& I_{1}(n) \leq c E^{t, \pi}\left[\exp \left(\mu \sum_{k=1}^{n-2} D\left(h_{k-1}, X_{\tau_{k}}-X_{\tau_{k-1}}\right)\right)\right. \\
& \quad \times E^{\tau_{n-2}, \pi_{n-2}}\left[\operatorname { e x p } \left(\mu D\left(h_{n-2}, X_{\tau_{n-1}}-X_{\tau_{n-2}}\right)\right.\right. \\
& \left.\left.\left.\quad+\left(\mu \underline{m}+\frac{(\mu \bar{\sigma})^{2}}{2}\right)\left(T-\tau_{n-1}\right)\right) 1_{\left\{\tau_{n-1}<T\right\}}\right] 1_{\left\{\tau_{n-2} \leq T\right\}}\right] \\
& \leq c^{2} E^{t, \pi}\left[\exp \left(\mu \sum_{k=1}^{n-2} D\left(h_{k-1}, X_{\tau_{k}}-X_{\tau_{k-1}}\right)\right)\right. \\
& \left.\left.\quad \times \exp \left(\left(\mu \underline{m}+\frac{(\mu \bar{\sigma})^{2}}{2}\right)\left(T-\tau_{n-2}\right)\right) 1_{\left\{\tau_{n-2} \leq T\right\}}\right]\right] \\
& \leq c^{n} \exp \left(\left(\mu \underline{m}+\frac{(\mu \bar{\sigma})^{2}}{2}\right)(T-t)\right),
\end{aligned}
$$

and therefore we see that

$$
\lim _{n \rightarrow \infty} I_{1}(n)=0
$$

On the other hand, since $1_{\left\{\tau_{n}>T\right\}}=\sum_{j=0}^{n-1} 1_{\left\{\tau_{j}<T \leq \tau_{j+1}\right\}}$, we have

$$
\begin{aligned}
& I_{2}(n)=E^{t, \pi}\left[\exp \left(\mu \sum_{k=1}^{n} D\left(h_{k-1}, X_{T \wedge \tau_{k}}-X_{\tau_{k-1}}\right) 1_{\left\{\tau_{k-1}<T\right\}}\right) 1_{\left\{\tau_{n} \geq T\right\}}\right] \\
& =E^{t, \pi}\left[\sum_{j=0}^{n-1} \exp \left(\mu \sum_{k=1}^{n} D\left(h_{k-1}, X_{T \wedge \tau_{k}}-X_{\tau_{k-1}}\right) 1_{\left\{\tau_{k-1}<T\right\}}\right) 1_{\left\{\tau_{j}<T \leq \tau_{j+1}\right\}}\right] \\
& =E^{t, \pi}\left[\sum _ { j = 0 } ^ { n - 1 } \operatorname { e x p } \left(\mu \sum_{k=1}^{j+1} D\left(h_{k-1}, X_{T \wedge \tau_{k}}-X_{\tau_{k-1}}\right) 1_{\left\{\tau_{k-1}<T\right\}}\right.\right. \\
& \left.\left.\quad+\mu \sum_{k=j+2}^{n} D\left(h_{k-1}, X_{T \wedge \tau_{k}}-X_{\tau_{k-1}}\right) 1_{\left\{\tau_{k-1}<T\right\}}\right) 1_{\left\{\tau_{j}<T \leq \tau_{j+1}\right\}}\right] .
\end{aligned}
$$


Noting that $\left\{\tau_{k}<T\right\} \cap\left\{T \leq \tau_{j+1}\right\}=\emptyset$ for all $k \geq j+1$, we have

$$
\begin{aligned}
& \exp \left(\mu \sum_{k=j+2}^{n} D\left(h_{k-1}, X_{T \wedge \tau_{k}}-X_{\tau_{k-1}}\right) 1_{\left\{\tau_{k-1}<T\right\}}\right) 1_{\left\{\tau_{j}<T \leq \tau_{j+1}\right\}} \\
= & \exp \left(\mu \sum_{k=j+2}^{\infty} D\left(h_{k-1}, X_{T \wedge \tau_{k}}-X_{\tau_{k-1}}\right) 1_{\left\{\tau_{k-1}<T\right\}}\right) 1_{\left\{\tau_{j}<T \leq \tau_{j+1}\right\}} \\
= & 1_{\left\{\tau_{j}<T \leq \tau_{j+1}\right\}}
\end{aligned}
$$

and that

$$
\begin{aligned}
\lim _{n \rightarrow \infty} I_{2}(n) & =\lim _{n \rightarrow \infty} E^{t, \pi}\left[\exp \left(\mu \sum_{k=1}^{n} D\left(h_{k-1}, X_{T \wedge \tau_{k}}-X_{\tau_{k-1}}\right) 1_{\left\{\tau_{k-1}<T\right\}}\right) 1_{\left\{\tau_{n} \geq T\right\}}\right] \\
& =\lim _{n \rightarrow \infty} E^{t, \pi}\left[\sum_{j=0}^{n-1} \exp \left(\mu \sum_{k=1}^{\infty} D\left(h_{k-1}, X_{T \wedge \tau_{k}}-X_{\tau_{k-1}}\right) 1_{\left\{\tau_{k-1}<T\right\}}\right) 1_{\left\{\tau_{j}<T \leq \tau_{j+1}\right\}}\right] \\
& =E^{t, \pi}\left[\sum_{j=0}^{\infty} \exp \left(\mu \sum_{k=1}^{\infty} D\left(h_{k-1}, X_{T \wedge \tau_{k}}-X_{\tau_{k-1}}\right) 1_{\left\{\tau_{k-1}<T\right\}}\right) 1_{\left\{\tau_{j}<T \leq \tau_{j+1}\right\}}\right] \\
& =E^{t, \pi}\left[\exp \left(\mu \sum_{k=1}^{\infty} D\left(h_{k-1}, X_{T \wedge \tau_{k}}-X_{\tau_{k-1}}\right) 1_{\left\{\tau_{k-1}<T\right\}}\right)\right] .
\end{aligned}
$$

Therefore, we obtain

$$
\begin{aligned}
& \lim _{n \rightarrow \infty} E^{t, \pi}\left[\exp \left(\mu \sum_{k=1}^{n} D\left(h_{k-1}, X_{T \wedge \tau_{k}}-X_{\tau_{k-1}}\right) 1_{\left\{\tau_{k-1}<T\right\}}+\mu W^{0}\left(\tau_{n}, \pi_{n}, h_{n}\right) 1_{\left\{\tau_{n} \leq T\right\}}\right)\right] \\
& =\lim _{n \rightarrow \infty} I_{1}(n)+I_{2}(n)=\exp (\mu W(t, \pi, h .)),
\end{aligned}
$$

having used Lemma 3.1. This completes the proof.

Lemma 5.7. The equality (3.47) holds.

Proof. By the definition of $\mathcal{A}^{n}$, the inclusions $\mathcal{A}^{n} \subset \mathcal{A}^{n+1} \subset \mathcal{A}$ hold for $n \geq 0$ and we have

$$
\sup _{h \in \mathcal{A}^{n}} W(t, \pi, h .) \leq \sup _{h \in \mathcal{A}^{n+1}} W(t, \pi, h .) \leq \sup _{h \in \mathcal{A}} W(t, \pi, h .) .
$$

From the definition of $W^{n}(t, \pi)$ and $W(t, \pi)$ it follows that

$$
W^{n}(t, \pi) \leq W^{n+1}(t, \pi) \leq W(t, \pi) .
$$

Therefore, from Lemma 5.5 we have

$$
\bar{W}^{n}(t, \pi) \leq \bar{W}^{n+1}(t, \pi) \leq W(t, \pi) .
$$

Thus, from Proposition 3.4 and (3.39), we obtain

$$
\bar{W}(t, \pi) \leq W(t, \pi) .
$$


On the other hand, for $h \in \mathcal{A}$

$$
\begin{aligned}
& \bar{W}^{n}(t, \pi)=W^{n}(t, \pi) \\
& \geq \frac{1}{\mu} \log E^{t, \pi}\left[\operatorname { e x p } \left(\mu \sum_{k=1}^{n} D\left(h_{k-1}, X_{T \wedge \tau_{k}}-X_{\tau_{k-1}}\right) 1_{\left\{\tau_{k-1}<T\right\}}\right.\right. \\
& \left.\left.+\mu W^{0}\left(\tau_{n}, \pi_{n}, h_{n}\right) 1_{\left\{\tau_{n} \leq T\right\}}\right)\right] .
\end{aligned}
$$

Letting $n \rightarrow \infty$ and applying Lemma 5.6,

$$
\bar{W}(t, \pi) \geq W(t, \pi, h .)
$$

and hence, we obtain

$$
\bar{W}(t, \pi)=W(t, \pi) .
$$

\section{References}

[1] R.Atar and O.Zeitouni, Exponential stability for nonlinear filtering. Ann. Inst. H.Poincare Probab. Statist. 33 697-725 (1996) Volume 71, Number 2 (2010), 371-399.

[2] T.Björk, M. H. A. Davis, C. Landén, Optimal investment under partial information, Math. Meth. Oper. Res. 71 (2010), 371-399.

[3] P. Bremaud Point processes and Queues: Martingale Dynamics. Springer Verlag, New York, 1981.

[4] J.Cvitanic, R.Liptser, B.Rozovski, A filtering approach to tracking volatility from prices observed at random times, The Annals of Applied Probability, 16 (2006), 1633-1652.

[5] J.Cvitanic, B. Rozovski and I. Zaliapin, Numerical estimation of volatility values from discretely observed diffusion data, Journal of Computational Finance, 9 (2006), 1-36.

[6] R.J. Elliott, L. Aggoun and J.B. Moore, Hidden Markov Models: Estimation and Control, Springer-Verlag New York (1995).

[7] R. Frey and W. Runggaldier, A nonlinear filtering approach to volatility estimation with a view towards high frequency data, International Journal of Theoretical and Applied Finance, 4 (2001), 199-210.

[8] K. Fujimoto, H. Nagai, W.J.Runggaldier, Expected log-utility maximization under incomplete information and with Cox-process observations. Preprint 2012.

[9] P. Gassiat, H.Pham and M. Sirbu Optimal investment on finite horizon with random discrete order flow in illiquid markets, International Journal of Theoretical and Applied Finance, 14 (2011), 17-40.

[10] P. Gassiat, F. Gozzi and H. Pham Investment/consumption problems in illiquid markets with regimes switching. Preprint (2011). 
[11] J. Grandell, Aspects of Risk Theory, Springer-Verlag New York (1991).

[12] F.Le Gland and N.Oudjane. Stability and Uniform Approximation of Nonlinear Filters Using The Hilbert metric, and Application to Particle Filters Annals of Applied Probability 14, 1 (2004), 144-187.

[13] C. Liverani, Decay of Correlations, Ann. of Math. (2), 142(2):239-301, 1995.

[14] K.Matsumoto Optimal portfolio of low liquid assets with a log-utility function. Finance and Stochastics, 10 (2006), 121-145.

[15] H. Nagai, Risk-sensitive quasi-variational inequalities for optimal investment with general transaction costs, Stochastic Processes and Applications to Mathematical Finance, ed. J. Akahori et al. (2007) 219-232

[16] H. Pham, Portfolio optimization under partial information: theoretical and numerical aspects. In: The Oxford Handbook on Nonlinear Filtering (D. Crisan and B. Rozovskii, eds.). Oxford University Press, (2011), 990-1018.

[17] H.Pham and P. Tankov, A model of optimal consumption under liquidity risk with random trading times, Mathematical Finance, 18 (2008), 613-627.

[18] H.Pham and P. Tankov, A coupled system of integrodifferential equations arising in liquidity risk models, Applied Mathematics and Optimization, 59 (2009), 147-173.

[19] L.C.G. Rogers and O. Zane, A simple model of liquidity effects. In: Advances in Finance and Stochastics, Essays in Honour of Dieter Sondermann (K.Sandmann and P.Schönbucher, eds.). Springer Verlag, pp. 161-176.

[20] M. Taksar and X.Zeng, Optimal terminal wealth under partial information: both the drift and the volatility driven by a discrete-time Markov chain. SIAM J. Control Optim. 46 (2007), no. $4,1461-1482$. 